CONTRACT TITLE AND NUMBER:

Bench-Scale Co-Processing

DE-AC22-87PC79818

CONTRACTOR NAME:

UOP

25 E. Algonquin Road

Des Plaines, I11 ino is 60017-5017
Date: March 7, 1990

Quarterly Report No. 6

Reporting Period 7/1/89-9/30/89

AUTHORS: Douglas A. Nafis

John G. Gatsis

Carl Lea

Mark A. Miller

CONTRACT PERIOD: Apri1 1, 1988 to March 31, 1991

$\mathrm{DOE} / \mathrm{PC} / 79818--\mathrm{T} 2$

QUARTERLY REPORT

DE91 000916

This quarterly report is the sixth report for contract $D E-A C 22-87 P C 79818$. The objective of this contract is to extend and optimize UOP's single-stage slurry-catalyzed co-processing scheme, which was developed under previous contract DE-AC22-84PC70002. Particular emphasis is given to defining and improving catalyst utilization and costs, evaluating alternative and disposable slurry-catalyst systems, and improving catalyst recycle and recovery techniques. The work during this quarter involved a series of bench-scale runs using a new Mo-based slurry catalyst. The results of bench-scale Runs 24 and 25 are discussed in the following report.

\title{
Run 24 Operations
}

Run 24 was the first bench-scale run to use Uop's new Mo-based slurry catalyst. A small batch of this catalyst, K6.2, was tested earlier in UOP's autoclave reactivity test and reported in Quarterly Report 3 for this contract [1]. Autoclave tests showed that the new Mo catalyst was 5 to 10 times more active than the $V$-based reference catalyst that was previously in use. A second larger batch of the Mo catalyst, K6.7, was prepared for bench-scale use. Run 24 was made with 0.13 wt $-\%$ of the KF. 7 catalyst, measured on a metal per moisture and ash free feed (MAFF) basis. Reference feedstocks (2:1 Lloydminster Vacuum Resid:Illinois Coal No. 6) were used, and the run was started at 
standard operating conditions $\left(425^{\circ} \mathrm{C}\right.$ and $\left.3,000 \mathrm{psig}\right)$. The reactor temperature was gradually increased over the course of the rur to determine the relationship between the conversions and temperature. Three test periods at each condition were worked up in detail. Periods 7,8 , and 11 were run at $427^{\circ} \mathrm{C}$; periods 21,22 , and 24 at $432^{\circ} \mathrm{C}$; and periods $33-35$ at $438^{\circ} \mathrm{C}$. Operating results from each of the test periods are summarized in Appendix A. Run 24 was shut down on August 6 (472 HOS) after the available feed was used up.

\section{Run 25 Operations}

Based on the results of Run 24, which failed to show the expected decline in the conversion of heptane insolubles above $435^{\circ} \mathrm{C}$ that had been observed in other runs, the decision was made to continue testing at even higher temperatures. A new batch of feed similar to that used for Run 24 was prepared. (The catalyst concentration, 0.11 wt-\% Mo, actually turned out to be slightly lower than that used in Run 24.) The temperature was increased from $429^{\circ} \mathrm{C}$ to $460^{\circ} \mathrm{C}$ over the course of the run. The bench-scale unit has never been operated stably at temperatures this high before. During test period 32 , the recycle pump packing blew out, and recycle flow was lost for about 40 minutes until the level of the high-pressure separator could be built back up. During this time, the reactor temperature reached an internal peak of about $485^{\circ} \mathrm{C}$. At about the same time, a major upset in the vacuum column occurred because of plugging in the reboiler recirculation line. Run 25 was eventually shut down on Sept. 7 (504 HOS), because of these problems.

An examination of the reactor following the run showed considerable coke buildup on the walls in the top 12 inches of the reactor. However, whether this buildup was due to the operation at higher-thannormal severities or whether it was due to the temperature excursion that resulted from loss of the recycle stream is uncertain. Subsequent runs (see Quarterly Report No. 7) have shown good operation at $460-470^{\circ} \mathrm{C}$ [2], which tend to support the latter explanation. Run 25 results are summarized in Appendix B. A complete set of plots of the 
Run 24 and 25 conversions, yielcs, and product properties vs. temperature is given in Appendix $C$.

\section{Discussion of Results}

Previous autoclave testing has demonstrated that equivalent conversions, yields, and product properties can be achieved with the new Mo-based catalyst using on? y about one-tenth of the metal concentration required for the former $V$-based reference catalyst [3]. This advantage was borne out in the bench-scale unit. As shown in Table 1, superior yields and product properties were achieved with 0.11 wt $-\%$ of Mo in kun 25 compared with 1.0 wt-\% $V$ in Run 21 . These results are compared at maximum nondistillables conversion, which for the new Mo catalyst was at a significantly higher temperature.

Autoclave tests with both $V$ and Mo catalysts have shown a decrease in MAF coal arid heptane insoluble conversions at temperatures above $425^{\circ} \mathrm{C}$. This 15 illustrated for the Mo catalyst in Figure 1 . Similar results were also observed in the bench-scale unit in Run 5 , in which a $v$-based reference catalyst was used [4]. The decline in conversions at high temperature has been attributed to retrograde reactions in which the asphaltenes that are formed by thermal break-up of the coal polymerize to form coke. In the presense of a catalyst and hydrogen, these reactive intermediates are preferentially converted to stable light products. However, at high temperatures, the rates of the retrogressive thermal reactions exceed those of the beneficial catalytic reactions, and pitch and coke are formed at the expense of more-valuable light products. Because coal conversion is measured by the disappearance of toluene insolubles and the formation of coke increases the measured toluene insolubles, the amount of "unconverted coal" actually increases at high temperatures as a result of coke formation.

Thus, it was surprising that the conversions in Runs 24 and 25 (Figure 2) showed an almost linear increase with temperature, with no signs of falling off, even at $460^{\circ} \mathrm{C}$. The unconverted coal (toluene 
insolubles less ash) showed a steady decrease without the characteristic upturn that is typically observed as a result of coking at higher temperatures (Figure 3). One major difference between Runs 24 and 25 and earlier temperature studies with $V$ catalyst (Runs 5 and 9 ) is the inclusion of a liquid recycle stream from the bottom of the highpressure separator to the reactor inlet. The space velocity was maintained constant between the runs, but the addition of the recycle stream (5:1 recycle:fresh feed) resulted in greater mixing and higher liquid velocities through the reactor. The improved high temperature conversions may be due to better contacting of the reactive coal fragments with hydrogen and catalyst (as a result of increased reactor back-mixing) or to decreased relative contact between the coke precursors and the hot reactor wa $17 \mathrm{~s}$ because of greater superficial liquid velocities in the reactor. Hydrodynamic differences resulting from the recycle may have also affected the flow regime, heat transfer characteristics, n. gas void fraction in the reactor. Further reactor modeling studies are required to thoroughiy understand these phenomena.

The extent of coking may also depend on the physical properties of the col.serted products: Petroleum resid and coal liquefaction products are complex mixtures of oils (heptane soluble and pentane soluble), resins (heptane soluble and pentane insoluble), and asphaltenes (heptane insoluble). Asphalteres have little or no solubility in the oils. The resins help to keep the asphaltenes dispersed (peptized) in the continuous oils by acting as an intermediate phase. Figure 4 gives a theoretical illustration of this dispersion [5]. The asphaltenes of higher aromaticity are surrounded by resin components of lower aromaticity, which can coexist with oils of the lowest aromaticity. The solubility of the asphaltenes depends greatly on their $H / C$ ratio, the number of aliphatic side chains that attach to the aromatic rings, and on the peptizing power of the resins, which is determined by the amount, aromaticity, and polarity of the resins. For the same aromaticity, resins with higher polarity (i.e., more heteroatoms) are better for peptizing the asphaltenes. At high severities, the aliphatic side chains are cracked from the asphaltenes and resins, and 
heteroatoms are removed, making the resins less polar. Both of these effects lead to reduced asphaltene solubility. When the asphaltenes can no longer remain peptized, they precipitate out, undergo further thermal condensation, and ultimately become coke. Thus, asphaltene solubility may play as much of a role in coking as the reaction kinetics.

Whatever the explanation, the improvement in pilot plant operability at high temperatures in Runs 24 and 25 represents an important process advance. The ability to operate at high nondistillable conversions; without significant carbon loss to retrograde reactions, significantly improves the economics of slurry co-processing. As shown in Figure 2, increasing the average reactor temperature by $30^{\circ} \mathrm{C}$ increases the conversion of nondistillables from about $55 \%$ to $85 \%$. Based on a correlation of product values to hydrogen index for a marker crude price of $\$ 17 /$ barrel [6], this increase in nondistillable conversion corresponds to an increase of about $\$ 4 /$ barre 1 in the value of the $\mathrm{C}_{5}+1$ iquid product. Although the yield of $\mathrm{C}_{5}+$ liquid product decreases from $86.2 \mathrm{w}^{+}-\%$ of MAF feed to $83.7 \%$ as a result of the increased production of ight ends, the total product value still increases by about $30 \%$ because of the improved product quality and increased by-product credits for sulfur. Table 2 compares the yields and product properties at $427^{\circ} \mathrm{C}, 446^{\circ} \mathrm{C}$, and $459^{\circ} \mathrm{C}$. Table 3 gives a simplified cost analysis for the three cases. Assuming that the higher temperature operation does not require more expensive metallurgy or fractionation (and that capital costs are, therefore, the same as the base case), operation with 0.12 wt-\% of Mo catalyst would be profitable at a marker crude price of $\$ 28 /$ barrel. 


\section{References}

1. D. A. Nafis, et a1., "Quarterly Report No. 3," prepared for the United States Department of Energy under Contract No. DE-AC22-87PC79818, Feb. 15, 1989.

2. D. A. Nafis, et al., "Quarterly Report No. 7," prepared for the United States Department of Energy under Contract No. DE-AC22-87PC79818, Unissued.

3. D. A. Nafis, et a1., "Quarterly Report 3."

4. J. G. Gatsis, et a1., "Coal Liquefaction Co-Processing -Topical Report No. 2," prepared for the United States Department of Energy under Contract No. DE-AC22-84PC70002, December 31, 1987, pp. 2-40.

5. D. E. Allan, et a1., "Visbreaking Gains Renewed Interest", Chemical Engineering Progress, pp.85-89 (Jan. 1983).

6. D. A. Nafis, et a1., "UOP Co-Processing Developments," Paper presented at the Pittsburgh Energy Technology Center Contractors' Review Meeting, Pittsburgh, PA, Oct. 2-4, 1989, p. 9 .

7. D. A. Nafis, et a1., "Coal Liquefaction Co-Processing Final Report, " prepared for the United States Department of Energy under Contract No. DE-AC22-84PC70002, Sept. 19, 1988. 
Table 1

Comparison of Bench-Scale Results for $0.11 \mathrm{wt}-\%$ Mo

\section{and $1.0 \mathrm{wt}-\% \vee \mathrm{Catalysts}$}

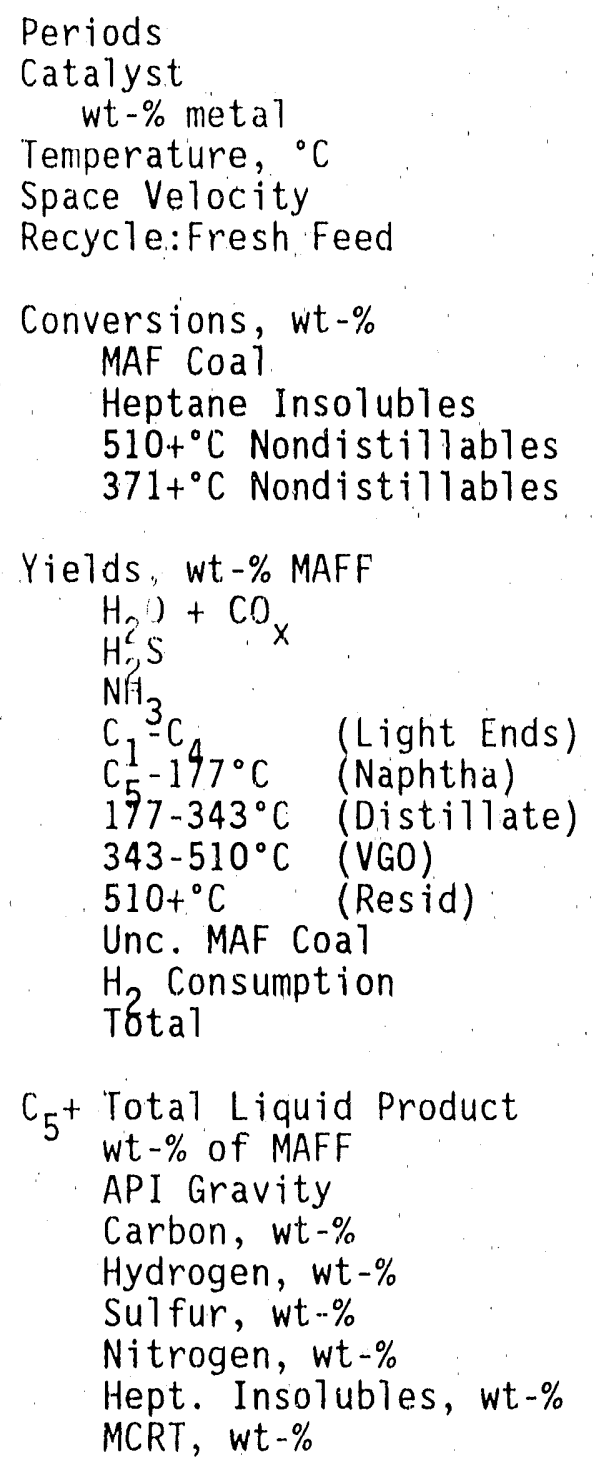

\begin{tabular}{rr} 
Run 21 & Run 25 \\
\hline $17-19$ & $\mathrm{~K} 6.41$ \\
$\mathrm{~K} 1.2$ & $0.11 \mathrm{Mo}$ \\
$1.0 \mathrm{~V}$ & 459.4 \\
425.8 & Base \\
Base & 5.5 \\
5.0 &
\end{tabular}

88.3

93.0

85.7

56.4

88.5

35.5

85.7

69.7

$5.9 \quad 4.3$

$\begin{array}{ll}1.5 & 3.5\end{array}$

$0.2 \quad 0.8$

$2.3 \quad 9.1$

$5.7 \quad 14.5$

$17.7 \quad 36.0$

$30.2 \quad 22.9$

$34.6 \quad 10.3$

$3.9 \quad 2.3$

$\frac{(2.1)}{100.0} \quad \frac{(3.6)}{100.0}$

$\begin{array}{rr}88.2 & 83.7 \\ 16.8 & 19.9 \\ 84.6 & 84.4 \\ 11.4 & 11.0 \\ 1.1 & 1.1 \\ 0.9 & 0.4 \\ 2.5 & 3.1 \\ 8.3 & 6.2\end{array}$

Basis: R10 Lloydminster Resid, C1.4 Illinois Coal No. 6; 2:1 Resid:MAF Coal, 3000 psig. 
Table 2

\section{Effect of Severity on Yields and Product Properties}

Run

Periods

Recycle:Fresh Feed

Yields, wt\% MAFF

$\mathrm{H}_{2} \mathrm{O}+\mathrm{CO}_{X}$

$\mathrm{H}_{2}^{2} \mathrm{~S}$

$\mathrm{NH}_{3}-\mathrm{C}$ (Light Ends)

$1_{5}-147^{\circ} \mathrm{C}$ (Naphtha)

$197-343^{\circ} \mathrm{C}$ (Disti11ate)

$343-510^{\circ} \mathrm{C}$ (VGO)

$510+^{\circ} \mathrm{C}$ (Resid)

Unc. MAF Coal

$\mathrm{H}_{2}$ Consumption

Total

$\mathrm{C}_{5}+$ Total Liquid Product

wt $-\%$ of MAFF

API Gravity

Carbon, wt-\%

Hydrogen, wt-\%

sulfur, wt-\%

Nitrogen, wt-\%

Hept. Insolubles, wt-\%

MCRT, wt-\%

Hydrogen Index *

$\mathrm{C}_{5}+$ Value, $\$ / \mathrm{bbl} \star *$

\begin{tabular}{rrr}
\multicolumn{3}{c}{ Temperature, ${ }^{\circ} \mathrm{C}$} \\
\hline 427.4 & $\frac{446.0}{25}$ & $\frac{459.4}{25}$ \\
2.4 & 11,13 & 31,41 \\
$7,8,11$ & 5.8 & 5.5 \\
5.7 & &
\end{tabular}

6.7

1.6

4.0

4.3

0.2

2.5

5.4

17.6

29.2

34.0

5.6

$\frac{(2.8)}{100.0}$

$\frac{(2.8)}{100.0}$

86.2

12.9

84.7

10.0

2.1

0.9

9.8

12.7

0.1142

9.3

86.3

83.7

17.0

84.0

10.3

1.4

0.8

9.1

11.2

0.1195

10.7

84.4

11.0

1.1

0.4

3.1

6.2

0.1285

13.2

Basis: 0.12 wt-\% K6.7 (Mo) Catalyst; $2: 1$ Resid:MAF Coal, R10 Lloydminster Resid, C1.4 111 inois Coal No. 6; 3000 psig; base space velocity.

*Hydrogen Index $=[(w t-\% H)-2 / 32 *(w t-\% S)-3 / 14 *(w t-\% N)] /(w t-\% C)$

**Product Value $=f($ Hydrogen Index, $\$ 17 / \mathrm{bbl}$ Crude $)[6]$ 
Table 3

Product Values and Feed Costs vs. Reactor Severity

Run

Periods

Product Values, \$/MT MAFF

Sul fur

Ammonia

Fuel Gas

Naphtha

Distillate

VGO

Resid

Total

Feed Costs, \$/MT MAFF

Coal

Resid

Hydrogen

Total

\begin{tabular}{ccc}
\multicolumn{3}{c}{ Temperature, ${ }^{\circ} \mathrm{C}$} \\
\hline$\frac{427.4}{24}$ & $\frac{446.0}{25}$ & $\frac{459.4}{25}$ \\
$7,8,11$ & 11,13 & 31,41
\end{tabular}

1.66

0.18

2.87

15.25

38.02 .

54.99

$\frac{29.90}{142.87}$

142.87

\subsection{2}

0.36

3.62

6.31

33.88

51.84

49.15

$\frac{21.28}{166.25}$

0.72

10.44

40.94

77.77

43.13

$\frac{9.06}{185.68}$

\begin{tabular}{lll}
13.81 & 13.79 & 13.79 \\
58.58 & 58.63 & 58.63 \\
20.12 & 20.84 & 25.87 \\
\hline 92.51 & $\frac{20.26}{98.29}$ &
\end{tabular}

$\begin{array}{lll}50.36 & -72.99 & 87.39\end{array}$

Product - Feed Margin, \$/MT MAFF

Operating Costs, \$/MT MAFF

Utilities

Capital-Related Costs *

Labor-Related Costs

Catalyst (Throwaway Basis)

Total

\subsection{3}

26.73

0.77

5.24

26.73

0.77

$\frac{16.59}{49.22}$

$\frac{16.59}{49.33}$

6.02

26.73

0.77

$-\frac{16.59}{50.11}$

$1.14 \quad 23.66$

37.28

Operating Profit, \$/MT MAFF

\section{Cost Basis:}

Illinois Coal No. 6 - \$35/MT AR

Marker Crude Price - $\$ 28 / \mathrm{BBL}$

Product Prices $=\%$ of marker crude:

$\begin{array}{ll}\text { Naphtha } & 1.15 \times \mathrm{MC}=\$ 32.2 / \mathrm{BBL} \\ \text { Distillate } & 1.05 \times \mathrm{MC}=\$ 2.4 / \mathrm{BBL} \\ \text { VGO } & 1.00 \times \mathrm{MC}=\$ 28.0 / \mathrm{BBL} \\ \text { Resid } & 0.50 \times \mathrm{MC}=\$ 14.0 / \mathrm{BBL}\end{array}$

Fuel Gas

Hydrogen

Sulfur

Ammonia

- $\$ 2.60 / \mathrm{MM}$ BTU

- $\$ 718.6 / \mathrm{MT}$

- $\$ 110 / M T$

- $\$ 90 / \mathrm{MT}$

$\mathrm{MoO}_{3}$

$\$ 3.60 / 1 \mathrm{~b}$

$\begin{array}{ll}\text { Power } & \$ 0.049 / \mathrm{kWh} \\ \text { Fuel } & \$ 2.607 / \mathrm{MM} \text { BTU } \\ 150 \# \text { Steam } & \$ 5.27 / \mathrm{M \#} \\ 600 \# \text { Steam } & \$ 7.91 / \mathrm{M \#} \\ \text { BFW-Cond } & \$ 0.18 / \mathrm{M \#} \\ \text { CW } & \$ 0.05 / \mathrm{MGal}\end{array}$

Direct Labor $\$ 13 / \mathrm{hr}$

Fringe $25 \%$ of $\mathrm{DL}$

Supervision $35 \%$ of $\mathrm{DL}$

G\&A Overhead $100 \%$

*Estimated Erected Cost for Co-Processing Unit for 2,474 MT/D of coal and 25,677 BPD of Resid is $\$ 165.4$ million [7].

Capital Charges e $28.5 \%$, Maintenance e $3 \%$, Ins. \& Taxes $02 \%$ of EEC/yr. 


\section{Conversions vs. Temperature}



- MAF Coal + HI * MCRT $\square$ 610C+ND

గí. 2 Catalyst, R8 Resid, C1.3 Coal 


\section{Conversions vs. Temp. Runs 24 and 25}

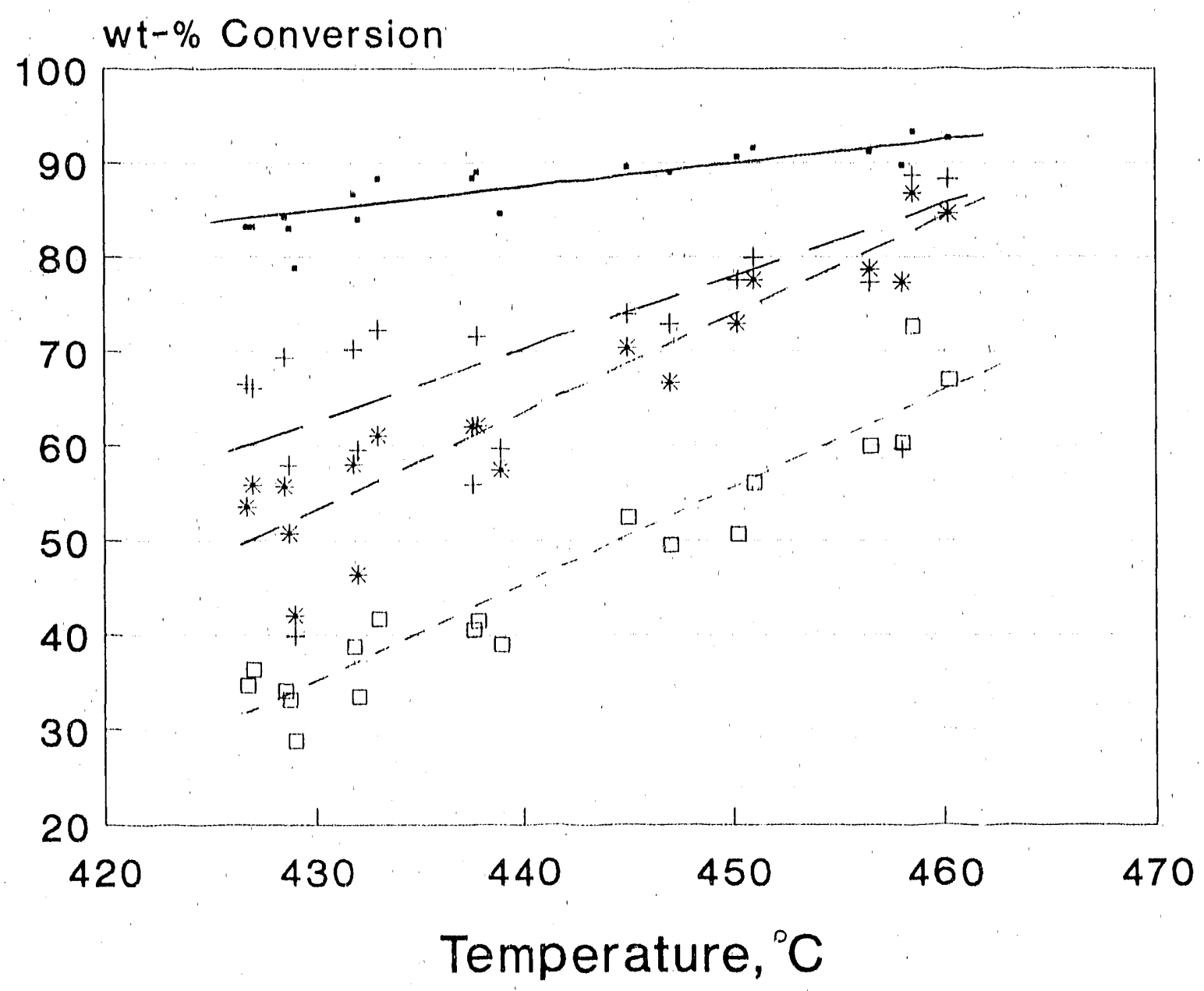

+ Heptane Insolubles

[1 371+C Non-distill.

K6.7 Catalyst $(0.11-0.13 \% \mathrm{Mo})$

C1.4 Coal, R10 Resid, 2:1 Resid:Coal 3000 psig, base WHSV 


\section{Unconverted MAF Coal vs Temperature}

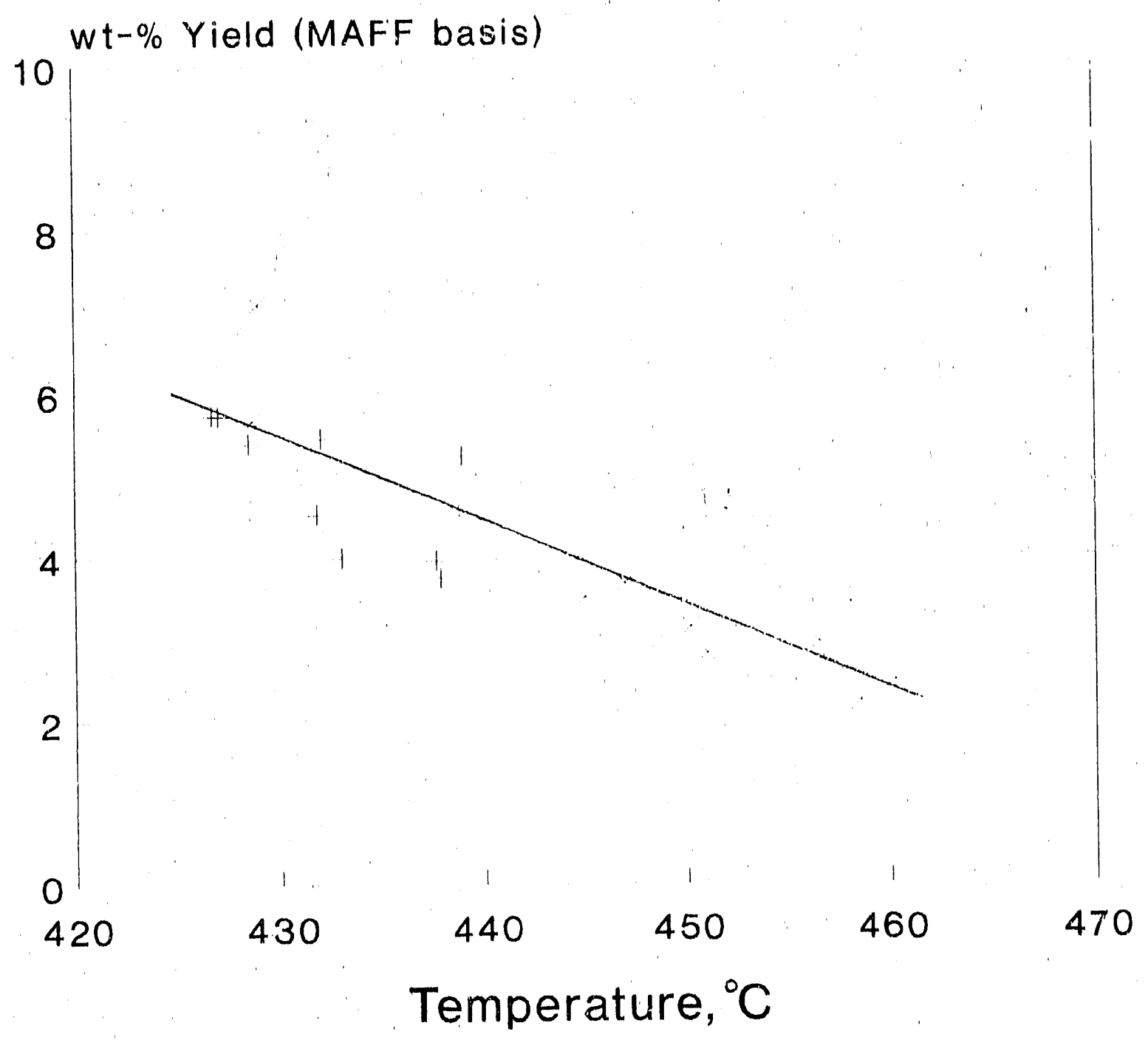

+ Run $24-0.13 \%$ Mo

Run $25-0.11 \%$ Mo

K6.7 Catalyst, C1.4 Coal, R10 Resid

3000 psig, base WHSV, 2:1 Resid:Coal 


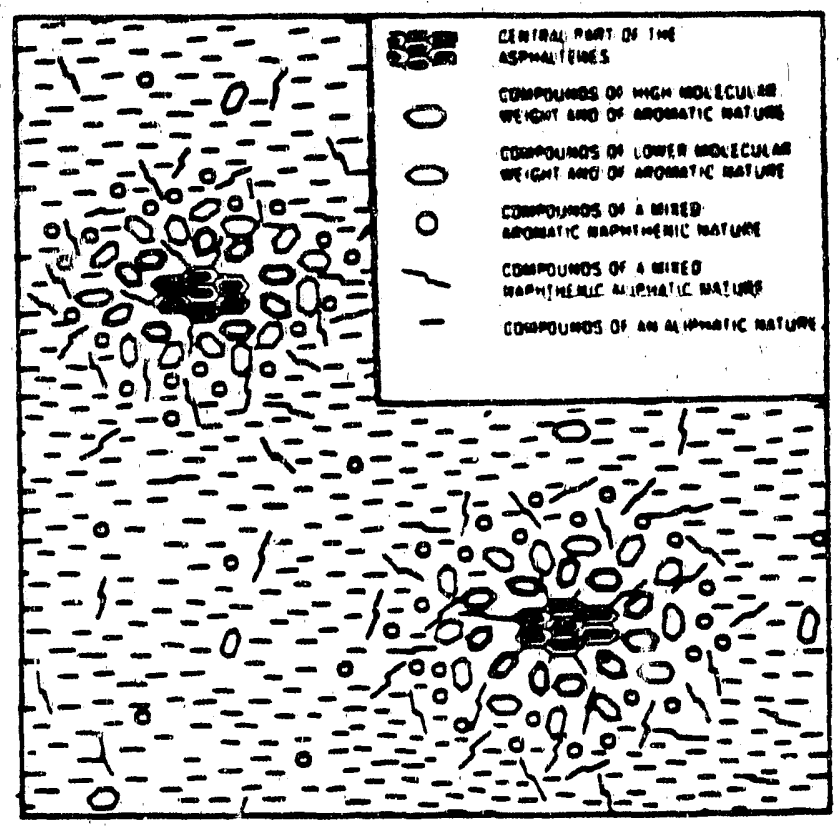


Run No. 24-2:1 Lloydminster Resid:Illinois Coal No. 6

\begin{tabular}{|c|c|c|c|c|c|c|c|c|c|c|c|}
\hline \multicolumn{12}{|c|}{ Run $\quad 0.13$ wt \%o Catalyst } \\
\hline Run : & 24 & 24 & 24 & 24 & 24 & 24 & 24 & 24 & 24. & 19 Ave. & 21 Ave. \\
\hline st Period & 7 & 8 & 11 & $21 \mathrm{~B}$ & 22 & 24 & 33 & 34 & 35 & LTOS & $17-19$ \\
\hline tial HOS & 84 & 96 & 1.32 & 280 & 292 & 316 & 424 & 436 & 448 & & \\
\hline Rx Int $T$, DegC & 427 & 426.7 & 428.5 & 432 & 431.8 & 433. & 437.8 & 438.9 & 437.6 & 425.5 & 425.8 \\
\hline tilet $P, p s i q$ & 3029 & 3025 & 3022 & 3026 & 3030 & 3036 & 3000 & 3006 & 3011 & 3058 & 3005 \\
\hline$x$ base & 0.91 & 1.09 & 0.97 & 1.01 & 0.98 & 1.06 & 0.97 & 1.33 & 0.88 & 1.00 & 1.11 \\
\hline Rec/Feed Ratio & 6.1 & 5.2 & 5.8 & 5.6 & 6.0 & 5.3 & 5.7 & 4.2 & 6.4 & NA & 5.0 \\
\hline d code & E11 & F11 & F11 & F11 & E11 & F11 & P11 & P11 & F11 & P08 & $\mathrm{POS}$ \\
\hline mS MAP feed & 1911.39 & 2297.71 & 2049.15 & 2135.86 & 2068.71 & 2234.61 & 2049,15 & $2809.66 \quad 1$ & 1848.38 & 5285.8 & 2348.4 \\
\hline sid Code & $R 10$ & R10 & R1: & R10 & R10 & RiO & R10 & $\mathrm{R} 10$ & R10 & $R O B$ & R10 \\
\hline Ins Resid & 1262.42 & 1517.58 & 1353.41 & 1410.68 & 1366.33 & 1475.90 & 1353.41 & 1855.71 & 1220.81 & 3475.6 & 1565.6 \\
\hline Code & $\mathrm{Cl} .4$ & $\mathrm{Cl} .4$ & $\mathrm{Cl} .4$ & 61.4 & $\mathrm{Cl} .4$ & $\mathrm{Cl} .4$ & $\mathrm{C1.4}$ & $\mathrm{C1.4}$ & C1.4 & 61.2 & $\mathrm{Cl} .4$ \\
\hline SMAP Coal & 648.97 & 780.13 & 695.74 & 725.18 & 702.38 & 758.71 & 695.74 & 953.95 & 627.57 & 1810.1 & 782.8 \\
\hline lyst code & K6.7 & R6.7 & R6.7 & $\mathbb{R} 6.7$ & $\mathbb{R} 6.7$ & $\mathrm{~K} 6.7$ & $\mathbb{R} 6.7$ & R6.7. & R6.7 & $\mathrm{K} 1.0$ & $\mathbb{R} 1.2$ \\
\hline cat. net./MAFF & 0.13 & $0.13^{\circ}$ & 0.13 & 0.13 & 0.13 & 0.13 & 0.13 & 0.13 & 0.13 & 1.00 & 0.9 \\
\hline \multicolumn{12}{|l|}{ Feed Distribution: } \\
\hline wt: C5-177C & 0.00 & 0.00 & 0.00 & 0.00 & 0.00 & 0.00 & 0.00 & 0.00 & 0.00 & 0.0 & \\
\hline $7-3436$ & 0.47 & 0.47 & 0.47 & 0.47 & 0.47 & 0.47 & 0.47 & 0.47 & 0.47 & 0.6 & 0.5 \\
\hline $510 \mathrm{C}$ & 11.31 & 11.31 & 11.31 & 11.31 & 11.31 & 11.31 & 11.31 & 11.31 & 11.3. & 13.0 & 11 \\
\hline$+c$ & 54.27 & 54.27. & 54.27 & 54.27 & 54.27 & 54.27 & 54,27 & 54.27 & 54.2 & 52.2 & 54 \\
\hline AF Coal & 33.95 & 33.95 & 33.95 & 33.95 & 33.95 & 33.95 & 33.95 & 33.95 & 33.9 & 34.2 & 33 \\
\hline wt: H2 Consump. & 2.5 .3 & 3.05 & 2.88 & 2.86 & 2.55 & 2.30 & 2.98 & 3.31 & 5.1 & 2.4 & \\
\hline ut: total feed & 102.53 & 103.05 & 102.88 & 102.86 & 102.55 & 102.30 & 102.98 & 103.31 & 105.13 & 102.4 & 102 \\
\hline \multicolumn{12}{|l|}{$\begin{array}{l}\text { Peed Analysis: } \\
\text { API gravity }\end{array}$} \\
\hline API gravity & -6 & -6 & -6 & -6 & -6 & -6 & -6 & -6 & -6 & -6.8 & 75. \\
\hline wt: carbon & 78.36 & 78.36 & 78.36 & 78.36 & 78.36 & 78.36 & 78.36 & 78.36 & 78.36 & 76.65 & 75. \\
\hline wt: hydrogen & 8.31 & 8.31 & 8.31 & 8.31 & 8.31 & 8.31 & 8.31 & 8.31 & 8.3 & 7.90 & \\
\hline sulfur & 4.13 & 4.13 & 4.13 & 4.13 & 4.13 & 4.13 & 4.13 & 4.13 & 4.1 & 4.38 & \\
\hline nitrogen. & 0.98 & 0.98 & 0.98 & 0.98 & 0.98 & 0.98 & 0.98 & 0.98 & 0.98 & 0.80 & \\
\hline hept. insol. & 8.63 & 8.63 & 8.63 & 8.63 & 8.63 & 8.63 & 8.63 & 8.63 & 8.63 & 8.49 & \\
\hline MCRI & 11.07 & 11.07 & 11.07 & 11.07 & 11.07 & 11.07 & 11.07 & 11.07 & 11.07 & 10.55 & \\
\hline & 0.68 & 0.68 & 0.68 & 0.68 & 0.68 & 0.68 & 0.68 & 0.68 & 0.68 & 0.96 & \\
\hline
\end{tabular}


Run No. 21 - 2:1 Lloydminster Resid:Illinois Coal No. 6 0.13 wt Yo Catalyst

\begin{tabular}{|c|c|c|c|c|c|c|c|c|c|c|c|}
\hline Run & 24 & 24 & 24 & 24 & 24 & 24 & 24 & 24 & 24 & 19 Ave. & 2.1 Ave. \\
\hline Test Period & 7 & 8 & 11 & $21 B$ & 22 & 24 & 33 & 34 & 35 & LMOS & $17-19$ \\
\hline \multicolumn{12}{|l|}{ Products: } \\
\hline Grams Cas COx & $3.50^{\circ}$ & 3.49 & 2.86 & 4.41 & 4.93 & 4.59 & 3.79 & 4.04 & 3.79 & 2.7 & 0.0 \\
\hline Grans Gas (1'. & 17.16 & 17.84 & 14.83 & 22.21 & 22.20 & .23 .04 & 34.87 & 28.32 & 26.42 & 45.7 & 17.2 \\
\hline Grams Gas C2 & 13.33 & 14.67 & 12.50 & 18.22 & 18.55 & 18.87 & 21.17 & 24.38 & 22.76 & 36.6 & 13.9 \\
\hline Grans Gas $\mathrm{C3}$ & 12.53 & 14.46 & 13.04 & 17.94 & 18.35 & 18.91 & 21.43 & 24.68 & 22.03 & 38.3 & 14.5 \\
\hline Grams Gas C4 & 7.92 & 9.07 & 8.11 & 14.69 & 13.17 & 12.06 & 13.35 & 16.74 & 11.73 & 22.9 & 7.7 \\
\hline Gramb Gas C5t & 9.24 & 11.78 & 11.01 & 9.73 & 10.77 & 16.43 & 17.14 & 17.97 & 13.02 & 26.9 & 6.5 \\
\hline Grams Gas NH3 & 3.30 & 4.51 & 3.69 & 6.25 & 5.97 & 6.32 & 9.49 & $6: 06$ & 4.80 & NA & 4.3 \\
\hline Grans Gas H2S & 25.22 & 28.59 & 32.14 & 41.11 & 41.04 & 43.50 & 35.66 & 52.86 & 43.45 & 35.4 & 36.1 \\
\hline Grams Net H2O & 154.6 & 113.0 & 132.0 & 93.9 & 68.7 & 76.8 & 92.0 & 92.0 & 63.0 & 282.8 & 137.7 \\
\hline Grans S w/1iet 1820 & 12.4 & NA & $\mathrm{NA}$ & 15.1 & 12.3 & 11.2 & MA & NA & NA & NA & $K A$ \\
\hline Grams OH Product & "657.00 & 872.00 & 785.00 & 614.00 & 764.00 & 927.00 & 765.00 & 1194.00 & 852.00 & 2308.7 & .1011 .3 \\
\hline Grans ISB Product & 946.55 & 1079.77 & 983.07 & 1156.98 & 1047.92 & 1038.57 & 816.48 & 1334.49 & 828.74 & 2439.6 & 1043.2 \\
\hline Grams Unconv.coal & 110.35 & 127.97 & 110.50 & 112.69 & 93.48 & 88.84 & 68.59 & 149.31 & 73.88 & 194.3 & 91.0 \\
\hline Grans $\mathrm{H}_{2}$ Consunp. & 48.31 & 70.07 & 59.07 & 61.08 & 52.74 & 51.32 & 61.05 & 92.94 & 94.91 & 124 & 48.2 \\
\hline Total (MAF) wt bal & 100.68 & 97.02 & 100.03 & 6.83 & 100.00 & 100.01 & 89.52 & 101.46 & 101.15 & 100.8 & 99.4 \\
\hline lotal C balarice & 96.97 & 97.31 & 98.02 & 96.59 & 100.16 & 99.88 & 89.91 & 104.83 & 105.03 & 101.5 & 99.0 \\
\hline Total H balance & 93.60 & 88.22 & 92.32 & 90.05 & $95.55^{\prime}$ & 96.52 & 86.23 & 92.08 & 83.70 & 106.6 & 106.2 \\
\hline Total S balance & 94.18 & 82.84 & 87.09 & 102.70 & 105.53 & 100.40 & 78.99 & 94.56 & 96.38 & 66.9 & 74.2 \\
\hline Total N balance* & 100.00 & 100.00 & 100.00 & 100.00 & 100.00 & 100.00 & 100.00 & 100.00 & 100.00 & 100.0 & 100.0 \\
\hline Total ash balance & 110.28 & 109.54 & 101,03 & 99.60 & 89.91 & 94.77 & 89.01 & 121.69 & 108.48 & 101.0 & 86.8 \\
\hline Total Cat. balance & 84.21 & 108.75 & 96.47 & 82.07 & 79.69 & 80.26 & 78.73 & 105.77 & 97.97 & 90.4 & 62.7 \\
\hline \multicolumn{12}{|c|}{ Norualized Conversions: } \\
\hline Coal & 83.11 & 83.09 & 84.12 & 83.95 & 86.69 & 88.29 & 88.99 & 84.57 & 88.36 & 89.3 & 88.3 \\
\hline C7 Insoluble & 66.05 & 66.44 & 69.29 & 59.57 & 70.21 & 72.25 & 71.56 & 59.68 & 55.97 & 79.3 & 85.7 \\
\hline Nondist. (510Ct) & 55.90 & 53.56 & 55.71 & 46.39 & 58.06 & 61.07 & 62.10 & 57.43 & 61.99 & 50.2 & 56.4 \\
\hline Dist. $(371 C+)$ & 36.22 & $34.5 \%$ & 33.94 & 33.33 & 38.66 & 41.63 & 41.38 & 38.88 & 40.42 & 34.9 & 35.5 \\
\hline \multicolumn{12}{|c|}{ Product Distribution: } \\
\hline wt: $\mathrm{H} 20+\mathrm{COX}$ & 8.22 & 5.23 & 6.58 & 4.75 & 3.56 & 3.64 & 5.22 & 3.37 & 3.57 & 5.3 & 5.9 \\
\hline wt: $\mathrm{H} 2 \mathrm{~S}$ & 1.89 & 1.28 & 1.57 & 2.76 & 2.62 & 2.48 & 1.94 & 1.35 & 2.32 & 0.6 & 1.5 \\
\hline$w_{t}^{0} \mathrm{NH3}$ & 0.17 & 0.20 & 0.18 & 0.30 & 0.29 & 0.28 & 0.52 & 0.21 & 0.26 & 0.4 & 0.2 \\
\hline wt: $\mathrm{Cl}-\mathrm{Cl}$ & 2.65 & 2.51 & 2.37 & 3.53 & 3.49 & 3.26 & 4.41 & 3.30 & 4.44 & 2.7 & 2.3 \\
\hline wt: C5-177C & 4.86 & 5.70 & 5.62 & 8.77 & 2.31 & 5.03 & 5.73 & 4.82 & 7.07 & 5.9 & 5.7 \\
\hline wt $177-343 \mathrm{C}$ & 17.24 & 18.81 & 16.68 & 13.07 & 24.06 & 24.40 & 21.70 & 23.60 & 22.87 & 18.2 & 17.7 \\
\hline wet: $343-510 \mathrm{C}$ & 28.53 & 28.34 & 30.82 & 22.42 & 29.25 & 28.88 & 30.03 & 28.59 & 31.07 & 31.5 & 30.2 \\
\hline wt $510+c$ & 33.17 & 35.23 & 33.68 & 41.85 & 32.49 & 30.37 & 29.70 & 32.32 & 29.58 & 34.2 & 34.6 \\
\hline wto Unc MAF Coal & 5.73 & 5.74 & 5.39 & 5.45 & 4.52 & 3.98 & 3.74 & 5.24 & 3.95 & 3.7 & 3.9 \\
\hline$\omega t:$ total prod & 102.57 & 103.05 & 102.88 & 102.91 & 102.59 & 102.33 & 102.98 & 103.31 & 105.13 & 102.4 & 102.1 \\
\hline
\end{tabular}


Run No. 24 - 2:1 Lloydininster Resid:Illinois Coal No. 6

0.13 wt? Ho Catalyst

\begin{tabular}{|c|c|c|c|c|c|c|c|c|c|c|c|}
\hline Run & 24 & 24 & 24 & 24 & 24 & 24 & 24 & 24 & 24 & 19 Ave. & 21 Ave. \\
\hline Test Period & 7 & 8 & 11 & $21 B$ & 22 & 24 & 33 & 34 & 35 & LITOS & $17-19$ \\
\hline \multicolumn{12}{|c|}{ Toluene Soluble Bottoms Product Properties: } \\
\hline TSB API & 3.3 & $3.4^{\circ}$ & 3.4 & 2.3 & 3.9 & 2.9 & 0.3 & 0.7 & 0.3 & 3.3 & 7.4 \\
\hline TSB wt: C & 85.62 & 85.33 & 85.34 & 86.45 & 85.56 & 86.16 & 86.29 & 85.75 & 86.47 & 85.98 & 88.3 \\
\hline TSB wt: H & 9.15 & 9.14 & 9.11 & 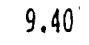 & 9.54 & 9.38 & 9.15 & 9.27 & 9.14 & 10.18 & 11.0 \\
\hline TSB wt: S & 2.39 & 2.73 & $\therefore 62$ & 2.04 & 2.26 & 2.14 & 2.24 & 2.45 & 2.04 & 2.27 & 1.6 \\
\hline TSB wt: N & 1.23 & 1.23 & .30 & 1.03 & 1.00 & 1.12 & 0,98 & 1.11 & 1.16 & NA & 1.6 \\
\hline TSB wt? HI & 17.96 & 17.88 & 16.24 & 21.27 & 16.32 & 17.07 & 19.02 & 25.76 & 33.71 & 11.65 & 4.9 \\
\hline TSB wt\} MCRT & 21.13 & 23.34 & 22.76 & 22.28 & 20.29 & 21.66 & 24.09 & 23.20 & 24.50 & $N A$ & 16.4 \\
\hline TSB wt? ash & 0 & 0 & 0 & 0 & 0 & 0 & 0 & 0 & 0 & 0.01 & 0.0 \\
\hline TSB ppiu Mo & 0 & 0 & 0 & 0 & 0 & 0 & 0 & 0 & 0 & 38 & 11.6 \\
\hline \multicolumn{12}{|l|}{ TSB D1160, vols } \\
\hline IBP, Deg C & $\mathrm{NA}$ & MA & NA & 372 & $\mathrm{HA}$ & 182 & 369 & $N A$ & 130 & 398.3 & 210.3 \\
\hline $5 \%$ & HA & MA & MA & 428 & NA & 392 & 430 & Nh & 415 & 457.3 & 467.0 \\
\hline $10 \%$ & NA & MA & NA & 454 & $N A$ & 432 & 461 & $N A$ & 445 & 473.3 & 481.3 \\
\hline $20 \%$ & NA & $N A$ & $N A$ & 487 & NA & 470 & 483 & NA & 481 & 493.0 & 504.0 \\
\hline $30 \%$ & $M A$ & MA & NA & NA & NA & 502 & 509 & NA & 511 & 512.8 & 527.3 \\
\hline $40 \%$ & NA & NA & NA & NA & $H A$ & 537 & NA & $\mathrm{NA}$ & 525 & $\mathrm{NA}$ & NA \\
\hline $50 \%$ & NA & NA & NA & $\mathrm{NA}$ & $N A$ & $\mathrm{MA}$ & $\mathrm{NA}$ & $N A$ & HA & $N A$ & MA \\
\hline $60 \%$ & NA & NA & NA & $N A$ & NA & NA. & $\mathrm{MA}$ & NA & NA & $N A$ & HA \\
\hline EP & NA & NA & NA & 503 & $N A$ & 551 & 532 & $N A$ & 525 & 542.8 & 539.7 \\
\hline$\%$ QEP & NA & NA & NA & 28 & $N A$ & 45 & 39 & $N A$ & 40 & MA & 36.3 \\
\hline wt: Dil60 OH & 41.32 & 39.3 & 39.97 & 28 & 43.8 & 45 & 38.7 & 37.08 & 39.6 & 42.42 & 36.3 \\
\hline TSB OH API & $\mathrm{NA}$ & $N A$ & NA & 11.5 & $N A$ & 11.7 & 10.3 & $\mathbb{N A}$ & 11.8 & 10.9 & 14.5 \\
\hline \multicolumn{12}{|l|}{ TSB OH GLC, wt? } \\
\hline IBP, Deg C & NA & NA & $N A$ & 273 & $N A$ & 237 & 286 & NA & 108 & 340.3 & 252.7 \\
\hline $5 \%$ & NA & $\mathrm{NA}$ & $\mathrm{NA}$ & 348 & $N A$ & 324 & 361 & $\mathbb{N A}$ & 340 & 412.0 & 403.3 \\
\hline $10 \%$ & NA & NA & NA & 374 & NA & 366 & 385 & $N A$ & 374 & 434.5 & 431.7 \\
\hline $20 \%$ & NA & $\mathrm{NA}$ & $\mathrm{NA}$ & 403 & $\mathrm{NA}$ & 408 & 415 & $N A$ & 408 & 459.0 & 459.0 \\
\hline $30 \%$ & NA & NA & $\mathrm{NA}$ & 423 & NA & 434 & 434 & $N A$ & 429 & 474.8 & 475.0 \\
\hline $40 \%$ & NA & $N A$ & $\mathrm{NA}$ & 438 & $N A$ & 454 & 449 & $N A$ & 446 & 488.8 & 487.0 \\
\hline $50 \%$ & NA & MA & NA & 451 & $\mathrm{NA}$ & 470 & 463 & $N A$ & 462 & 500.3 & 497.7 \\
\hline $60 \%$ & NA & $\mathrm{NB}$ & NA & 465 & NA & 483 & 473 & $\mathrm{NA}$ & 475 & 511.8 & 508.0 \\
\hline $70 \%$ & $\mathrm{NA}$ & $\mathrm{NA}$ & $N A$ & 477 & $N A$ & 499 & 484 & NA & 488 & 523.5 & 519.3 \\
\hline $80 \%$ & NA & NA & NA & 491 & $N A$ & 515 & 499 & $\mathbb{N A}$ & 503 & 536.5 & 531.0 \\
\hline $90 \%$ & NA & $\mathrm{NA}$ & $\mathrm{NA}$ & 510 & $N A$ & 535 & 520 & $N A$ & 523 & 554.5 & 545.3 \\
\hline $95 \%$ & NA & $N A$ & $N A$ & 526 & $N A$ & 549 & 535 & $N M$ & 537 & 568.5 & 559.7 \\
\hline$E P$ & NA & $\mathrm{NA}$ & NA & 562 & $N A$ & 574 & 567 & MA & 565 & 577.5 & 572.0 \\
\hline$\approx \operatorname{aEP}$ & NA & $N A$ & $\mathbb{N A}$ & 99.5 & $\mathrm{HA}$ & 99 & 99.5 & NA. & 100 & 100.0 & 97.3 \\
\hline wts D1160 Btms & 58.68 & 60.7 & 60.03 & 72 & 56.2 & 55 & 61.3 & 62.92 & 60.4 & 57.6 & 63.7 \\
\hline TSB Btns API & -2.2 & -4.9 & -5.4 & -2.8 & -1.7 & -5.8 & -6.4 & -5.6 & -6.7 & -4.0 & 5.7 \\
\hline
\end{tabular}


Run No. $24-2: 1$ Lloydminster Residitllinois Coal No. 6

\begin{tabular}{|c|c|c|c|c|c|c|c|c|c|c|c|}
\hline \multicolumn{12}{|c|}{0.13 wt $M_{0}$ Catalyst. } \\
\hline Run & 24 & 24 & 24 & 24 & 24 & 24 & 24 & 24 & 24 & \multirow{2}{*}{\multicolumn{2}{|c|}{$\begin{array}{l}19 \text { Ave. 21. Ave. } \\
\text { LTOS 17-19 }\end{array}$}} \\
\hline Test Period & 7 & 8 & 11 & 21B & 22 & 24 & 33 & 34 & 35 & & \\
\hline \multicolumn{12}{|c|}{$\begin{array}{l}\text { Test Period } \\
\text { Overhead Product Properties: }\end{array}$} \\
\hline OH API & 24.9 & 24.9 & 26.2 & 29.6 & 20.9 & 24.3 & 25.1 & 24.5 & 25.9 & 29.4 & 26.4 \\
\hline 애 wht's C & 84.47 & 83.72 & 83.22 & $8^{\prime \prime}, 66$ & 83.95. & 83.06 & 85.17 & 85.85 & 84.48 & HA & 84.8 \\
\hline OH wt: H & 11.1 & 11.18 & 11.36 & 11.63 & 11.21 & 10.93 & 11.56 & 11.4 & 11.52 & NA & 11.8 \\
\hline OH wet: 5 & $1.4 ?$ & $j .61$ & 1.55 & 1.39 & 1.43 & $1.3 i$ & 1.18 & i.17 & 1.2 & 1.44 & 0.7 \\
\hline OH wt: $\mathrm{H}$ & 0.4 & 0.412 & 0.331 & 0.367 & 9.474 & 0.423 & 0.435 & 0.43 & 0.416 & 0.33 & 0,2 \\
\hline \multicolumn{12}{|l|}{$\begin{array}{l}\text { OH wt? N } \\
\text { OH GIC, wt? }\end{array}$} \\
\hline IBP, Deg $C_{1}$ & 3 & 19 & 20 & -16 & 141 & 16 & 0 & 4 & 0 & 38.0 & -3.3 \\
\hline $5 \%$ & 114 & 112 & 104 & 49 & 188 & 106 & 87 & 111 & 75 & $12 n .0$ & 89.3 \\
\hline $10 \%$ & 164 & 166 & 165 & 79 & 211 & 175 & 166 & 183 & 147 & 103.3 & $15 \%, 3$ \\
\hline $20 \%$ & 218 & 222 & 226 & 105 & 242 & 223 & 225 & 228 & 219 & 219.8 & 233.3 \\
\hline $30 \%$ & 252 & 256 & 263 & 193 & 268 & 253 & 257 & 258 & 252 & 261.0 & 273.0 \\
\hline 408 & 282 & 287 & 298 & 252 & 293 & 280 & 285 & 285 & 283 & 298.0 & 306.0 \\
\hline $50 \%$ & 312 & 317 & 330 & 285 & 315 & 304 & 311 & 309 & 310 & 332.0 & 335.3 \\
\hline 608 & 341 & $345^{\circ}$ & 359 & 314 & 337 & 328 & 337 & 333 & 337 & 361.0 & 362.7 \\
\hline $70 \%$ & 369 & 373 & 387 & 344 & 361 & 354 & 363 & 358 & 364 & 388.3 & 388.7 \\
\hline $80 \%$ & 397 & 401 & 416 & 375 & 386 & 381 & $38 B$ & $38 ?$ & $39 i$ & $415.0^{\prime}$ & 414.0 \\
\hline $90 \%$ & 429 & 431 & 449 & 410 & 415 & 411 & 418 & 414 & 425 & 442.8 & 440.0 \\
\hline $95 \%$ & 449 & 452 & 476 & 429 & 431 & 429 & 434 & 432 & 445 & 460.5 & 455.0 \\
\hline EP & 505 & 507 & 519 & 464 & 467 & 476 & 469 & 460 & 474 & 488.5 & 480.7 \\
\hline  & 99.5 & 100 & 100 & 100 & 100 & 100 & 100 & 100 & 100 & 100.0 & 100.0 \\
\hline \multicolumn{12}{|c|}{ Total Liquid Properties } \\
\hline API & 12.1 & 13.0 & 13.5 & 11.8 & 11.1 & 13.0 & 12.3 & 11.9 & 13.3 & 13.6 & 16.8 \\
\hline wt? C & 85.1 & 84.6 & 84.4 & 85.5 & 84.9 & 84.7 & 85.7 & 85.8 & 85.5 & $N A$ & 86.6 \\
\hline wt: $H$ & 4.9 & 10.1 & 10.1 & 10.2 & 10.2 & 10.1 & 10.3 & 10.3 & 10.3 & $N A$ & 11.4 \\
\hline wt? $s$ & 2.0 & 2.2 & 2.1 & 1.8 & 1.9 & 1.8 & 1.7 & 1.8 & 1.6 & 1.9 & 1.2 \\
\hline$w t: N$ & 0.9 & 0.9 & 0.9 & 0.8 & 0.8 & 0.8 & 0.7 & 0.8 & 0.8 & NA & 0.9 \\
\hline wt? HI & 10.6 & 9.9 & 9.0 & 13.9 & 9.4 & 9.0 & 9.8 & 13.6 & 16.6 & 6.0 & 2.5 \\
\hline wto MCRT & 12.5 & 12.9 & 12.7 & 14.6 & 11.7 & 11.4 & 12.4 & 12.2 & 12.1 & $M A$ & 8.3 \\
\hline wt' dist. (371C-) & 30.9 & 32.3 & 30.2 & 29.1 & 35.6 & 38.6 & 36.8 & 37.5 & 38.6 & 31.9 & 31.6 \\
\hline \multicolumn{12}{|c|}{ Unoonverted Coál Solids Propertiesk»! } \\
\hline UCC wt: C & $84.8^{\circ}$ & 84.9 & 85.2 & 85.9 & 84.5 & 83.4 & 82.7 & 82.9 & 83.5 & $\mathbb{N A}$ & 82.2 \\
\hline UCC wt: $H$ & 5.9 & 6.2 & 6.5 & 6.1 & 6.2 & 5.9 & 5.7 & 5.8 & 5.7 & $N A$ & 5.7 \\
\hline UCC wt: S & 7.1 & 6.9 & 6.2 & 5.9 & 7.1 & 8.6 & 9.4 & 9.2 & 8.8 & HA & 10.2 \\
\hline UCC wt: N & 2.2 & 2.1 & 2.1 & 2.1 & 2.1 & 2.0 & 2.2 & 2.2 & 2.1 & HA & 1.8 \\
\hline 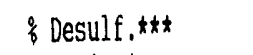 & 60.99 & 55.85 & 56.87 & 64.91 & 61.00 & 64,03 & 68.91 & 61.22 & 65.77 & 64.6 & 76.7 \\
\hline$\xi$ Denitrif, $* * *$ & 26.46 & 27.71 & 25.86 & 34.58 & 32.84 & 31.36 & 45.48 & 29.83 & 29.80 & $M A$ & 22.3 \\
\hline
\end{tabular}


Run No. 25 - 2:1 Lloydminster ResidiIllinols Coal No. 6

\begin{tabular}{|c|c|c|c|c|c|c|c|c|c|c|c|c|}
\hline \multirow[b]{2}{*}{ Run } & \multicolumn{2}{|c|}{ No Catalyst } & \multirow[b]{2}{*}{25} & \multirow[b]{2}{*}{25} & \multirow[b]{2}{*}{25} & \multirow[b]{2}{*}{25} & \multirow[b]{2}{*}{25} & \multirow[b]{2}{*}{25} & \multirow[b]{2}{*}{25} & & \multirow{3}{*}{$\begin{array}{l}19 \text { Ave. } \\
\text { LTOS }\end{array}$} & \multirow{3}{*}{$\begin{array}{l}21 \text { Ave, } \\
17-19\end{array}$} \\
\hline & 25 & 25 & & & & & & & & 25 & & \\
\hline I'est Period & 6 & 7 & 11 & 13 & 17 & 21 & 23 & 27 & 31 & 41 & & \\
\hline Initial yOS & 72 & 84 & 132 & 156 & 204 & 252 & 276 & 324 & 372 & 492 & & \\
\hline Ave $R x$ Int $T_{1}$ DegC & 429 & 428.7 & 445 & 447 & 450.2 & 451 & 458 & 456.5 & 460.2 & 458.5 & 425.5 & 425.8 \\
\hline Rx Outlet $P$, psig & 3008 & 3007 & 3010 & 3013 & 3014 & 3000 & 3000 & 3000 & 3000 & 3000 & 3058 & 3005 \\
\hline WHSV, $x$ base & 0.93 & 0,96 & 0.83 & 1.11 & 1.16 & 1.05 & 0.86 & 1.12 & 1.00 & 1.10 & 1.00 & 1.11 \\
\hline HISS Rec/Feed Ratio & 6,0 & 6.0 & 6.5 & 5.1 & 5.1 & 5.5 & 6.7 & 5.1 & 6.0 & 5.0 & $N A$ & 5.0 \\
\hline ed Code & F12 & $F 12$ & $\mathrm{~F} 12$ & $P 12$ & $\mathrm{~F} 12$ & F12 & F.12 & F12. & F12 & $\mathrm{F} 12$ & $\mathrm{FOB}$ & P09 \\
\hline ans MAF feed & 1971.20 & 2026.62 & 1758.12 & 2336.20 & 2447.99 & 2216.76 & 1817.36 & 2363.91 & 2103.06 & 2328.55 & $5285,8^{\circ}$ & 2348.4 \\
\hline sid code & R10 & R10 & R10 & R10 & R10 & R10 & R10 & R10 & R10 & R10 & ROB & $R 20$ \\
\hline ans Resid & 1313.92 & 1350.86 & 1171.90 & 1557.22 & 1631.74 & 1477.61 & 1211.38 & 1575.69 & 1401.82 & 1552.13 & 3475.6 & 1565.6 \\
\hline al code & 01.4 & $\mathrm{Cl} .4$ & $\mathrm{Cl} . \mathrm{d}$ & $\mathrm{Cl}, 4$ & $\mathrm{Cl} .4$ & $\mathrm{Cl} .4$ & $\mathrm{Cl} .4$ & $\mathrm{Cl} .4$ & 01.4 & $\mathrm{Cl} .4$ & $\mathrm{Cl} .2^{\prime}$ & $\mathrm{Cl}, 4$ \\
\hline IIS MAF Coal & 657.27 & 675.75 & 586.22 & 778.98 & 816.25 & 739.15 & 605.98 & 788.22 & 701.24 & 776.43 & 1810.1 & 782.8 \\
\hline yst code & K6.7 & K6.7 & K6.7 & K6.7 & $\mathrm{R} 6.7$ & K6.7 & $\mathrm{K} 6.7$ & K6.7 & K6.7 & $\mathrm{K} 6.7$ & $\mathrm{~K} 1.0$ & $\mathrm{~K} 1.2$ \\
\hline wt: cat. net./MAFF & 0.11 & 0.11 & 0.11 & 0.11 & 0.11 & 0.11 & 0.11 & 0.11 & 0.11 & 0.11 & 1.00 & 0.99 \\
\hline \multicolumn{13}{|l|}{ Peed Distribution: } \\
\hline wt? C5-177C & 0.00 & 0.00 & 0.00 & 0.00 & 0.00 & 0.00 & 0.00 & 0.00 & 0.00 & 0.00 & 0.0 & 0.0 \\
\hline wt? $177-343 C$ & 0.47 & 0.47 & 0.47 & 0.47 & 0.47 & 0.47 & 0.47 & 0.47 & 0.47 & 0.4 & 0.6 & 0.5 \\
\hline$w t \frac{6}{6} 343-510 C$ & 11.41 & 11.11 & 11.41 & 11,41 & 11.41 & 11.41 & 11.41 & 11.41 & 11.41 & 11.4 & 13.0 & 11.4 \\
\hline wet? $510+c$ & 54.77 & 54.77 & 54.77 & 54.77 & 54.77 & 54.77 & 54.77 & 54.77 & 54.77 & 54.7 & 52.2 & 54.8 \\
\hline wt: MAF coal & 33.34 & 33.34 & 33.34 & 33.34 & 33.31 & 33.34 & 33.34 & 33.34 & 33.34 & 33.3 & 34.2 & 33.3 \\
\hline wt? H2 Consurip. & 2.59 & 2.61 & 3.25 & 2.51 & 3.37 & 2.70 & 3.46 & 2.82 & 3.38 & 3.73 & 2.4 & 2.1 \\
\hline wts total feed & 102.59 & 102.61 & 103.25 & 102.51 & 103.37 & 102.70 & 103.46 & 102.82 & 103.38 & 103.73 & 102.4 & 102.1 \\
\hline \multicolumn{13}{|c|}{ 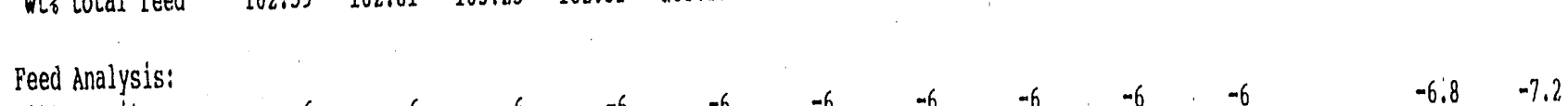 } \\
\hline API gravity & -6 & -6 & -6 & -6 & -6 & -6 & -6 & -6 & -6 & -6 & -6.8 & -7.2 \\
\hline wet carbon & 78.38 & 78.38 & 78.38 & 78.38 & 78.38 & 78.38 & 78.38 & 78,38 & 78.38 & 78.38 & 76.65 & 75.72 \\
\hline wts hydrogen & 8.32 & 8.32 & 8.32 & 8.32 & 8.32 & 8.32 & 8.32 & 8.32 & 8.32 & 8.3 & 7.90 & 8.03 \\
\hline wt: sulfur & 4.13 & 4.13 & 4.13 & 4.13 & 4.13 & 4.13 & 4.13 & $4.1 ?$ & 4.13 & 4.13 & 4.38 & 3.99 \\
\hline wt: nitrogen & 0.98 & 0.98 & 0.98 & 0,98 & 0.98 & 0.98 & 0.98 & 0.98 & 0.98 & 0.98 & 0.80 & 0.94 \\
\hline wt' hept, insol. & 8.64 & 8.64 & 8.64 & 8,64 & 8.64 & 8.64 & 8.64 & 8.64 & 8.64 & 8.64 & 8.49 & 8.34 \\
\hline wts MCRT & 11.08 & 11.08 & 11.08 & 11.08 & 11.08 & 11.08 & 11.08 & 11.08 & 11.08 & 11.08 & 10.55 & 10.70 \\
\hline wt? dist. (371c-- & 0.68 & 0.68 & 0.68 & 0.68 & 0.68 & 0.68 & 0.68 & 0.68 & 0.68 & 0.68 & 0.96 & 0.66 \\
\hline
\end{tabular}


Run No. $25-2: 1$ Lloydminster Resid:Illinois coal No. 6

0.11 wto Mo Catalyst

\begin{tabular}{|c|c|c|c|c|c|c|c|c|c|c|c|c|}
\hline Run & 25 & 25 & 25 & 25 & 25 & 25 & 25 & 25 & 25 & 25 & 19 Ave. & \\
\hline Test Period & 6 & 7 & 11 & 13 & 17 & 21 & 23 & 27 & 31 & 41 & LTOS & $17 \%$ \\
\hline \multicolumn{13}{|l|}{ Products: } \\
\hline Grams Gas COx & 3.82 & 3.81 & 4.22 & 3.56 & 4.83 & 4.81 & 2.88 & 6.30 & 3.97 & 4.74 & 2.7 & \\
\hline Grams Gas Cl & 20.00 & 20.91 & 32.63 & 33.15 & $39: 65$ & 41.22 & 42.65 & 53.93 & 52.47 & 52.57 & 45.7 & \\
\hline Grams Gas C2 & $16.4^{\prime} 7$ & 16.59 & 26.85 & 29.74 & 34.88 & 35.94 & 40.20 & 52.60 & 47.51 & 49.35 & 36.6 & \\
\hline Grans Gas C3 & 15.78 & 15.89 & 27.59 & 31.25 & 35,72 & 39.09 & 48.71 & 61.68 & 56.48 & 57.70 & 38.3 & \\
\hline Grams Gas C4 & 10.49 & 10.56 & 15,97 & 18.52 & 19.20 & 21.86 & 35.63 & 40.37 & 47.40 & 48.56 & 22.9 & \\
\hline Grans Gas C5t & 16.00 & 14,45 & 23.50 & $32: 03$ & 29.17 & 22.98 & 34.81 & 34.87 & 44.98 & 65.76 & 26.9 & \\
\hline Grans Gas NH3 & 2.07 & 4.85 & 6.53 & 9.82 & 7.96 & 7.77 & 7.77 & 12.40 & 16.69 & 19.19 & $N A$ & \\
\hline Grans Gas H2S & 40.93 & 41.49 & 44.49 & 50.67 & 55.91 & 51.76 & $4^{5}, 03$ & 58.72 & 56.98 & 75.54 & 35.4 & \\
\hline Grams Net $\mathrm{H} 20$ & 33.3 & .118 .1 & 80.0 & 67.1 & 10.8 & 65.9 & 13.7 & 119.4 & 104.3 & 80.7 & 282.8 & \\
\hline Grans S W/Net H2O & 7.7 & 7.9 & 15.0 & 18.9 & 19.2 & 16.1 & 17.3 & 17.6 & 14.7 & 9.3 & NA & \\
\hline Grams of Product & 730.00 & 713.00 & 957.00 & 1113.00 & 1358.00 & 1195.00 & 1077.00 & 1382.00 & 1118.00 & 1417.00 & 2308.7 & \\
\hline Grans ISB Product & 1014.35 & 1032.81 & 522.00 & 798.20 & 818.00 & 560.54 & 408.49 & 498.20 & 689.84 & 475.17 & 2439.6 & \\
\hline Grams Unconv.coal & 141.88 & 117.02 & 61.62 & 81.93 & 75.87 & 57.99 & 60.67 & 68.83 & 55.13 & 51.49 & 194.3 & \\
\hline Grans H2 Consump: & 51.14 & 52.80 & .57 .16 & 58.66 & 82.62 & 59,80 & 62.82 & 66.71 & 71.13 & 86.76 & 124 & \\
\hline Total (MAF) wt bal & 101.51 & 101.83 & 100.12 & 95.53 & 99.15 & 93.17 & 97.59 & 99,02 & 106.18 & 99.66 & 100.8 & \\
\hline Total C balance & 102.76 & 99.27 & 96.20 & 94.29 & 103.58 & 94,38 & 100.25 & 97.37 & 102.37 & 97.72 & 101.5 & \\
\hline Total H balance & 92.71 & 93.93 & 92.50 & 94,65 & 91.21 & 90.77 & 90.86 & 98.14 & 105.52 & 97.05 & 106.6 & \\
\hline Total S balance & 109.64 & 103.77 & 109.72 & 100.85 & 104,81 & 98.38 & 107.58 & 102.33 & 100.29 & 111.33 & 66.9 & \\
\hline Total N balancek & 100.00 & 100.00 & 100.00 & 100.00 & 100.00 & 100.00 & 190.00 & 100.00 & 100.00 & 100.00 & 100.0 & \\
\hline Total ash balance & 130.11 & 109.06 & 101.58 & 109.92 & 92.85 & 77.73 & 106.98 & 96,87 & 87.58 & 82.03 & 101.0 & \\
\hline Total cat, balance & 122.63 & 88.90 & 88.53 & 91.97 & 83.73 & 71.99 & 112.23 & 98.15 & 85.64 & 108.03 & 90.4 & \\
\hline \multicolumn{13}{|c|}{ Nornalized Conversions: } \\
\hline Coal & 78.73 & 82.99 & 89.50. & 88.99 & 90.63 & 91.58 & 89.74 & 91.18 & 92.59 & 93.35 & 89.3 & \\
\hline C7 Insoluble & 39.80 & 57.91 & 73.88 & 72.87 & 77.52 & 79.93 & $59.6 B$ & 77.25 & 88.25 & 88.70 & 79.3 & \\
\hline Nondist, (510Ct) & 42.00 & 50.73 & 70.36 & 66.63 & 72.93 & 77.52 & 77.22 & 78.61 & 84.56 & 86.82 & 56.2 & \\
\hline Dist. (371Ct) & 28.71 & 33,02 & 52.38 & 49.43 & 50.62 & 56.07 & 60.21 & 59.89 & 66.86 & 72.57 & 34.9 & \\
\hline \multicolumn{13}{|c|}{ Product Distribution: } \\
\hline wt: $\mathrm{H} 20+\mathrm{COX}$ & 1.85 & 5.91 & 4.78 & 3.17 & 0.64 & 3.42 & 0.93 & 5.37 & 4.85 & 3.68 & 5.3 & \\
\hline wt: $\mathrm{H} 2 \mathrm{~S}$ & 2.46 & 2.42 & 3.43 & 3.17 & $3.14^{\circ}$ & 3,33 & 3.58 & 3.31 & 3.25 & 3.68 & 0.6 & \\
\hline wt: NH3 & 0.10 & 0.2 .4 & 0.37 & 0.44 & 0.33 & 0.38 & 0.44 & 0.53 & 0.75 & 0.83 & 0.4 & \\
\hline wt? $\mathrm{Cl}-\mathrm{CA}$ & 3.14 & 3.10 & 5.85 & 5.05 & $5.3 \mathrm{~s}$ & 6.69 & 9.43 & 8.91 & 9.13 & 8.97 & 2.7 & \\
\hline wto $c 5-177 C$ & 4.41 & 4.06 & 11.32 & 12.70 & 3.22 & 3.02 & 9.43 & 3.56 & 13.23 & 15.72 & 5.9 & \\
\hline wet? $177-343 \mathrm{C}$ & 16.10 & 16.67 & 25.01 & 22.95 & 34.47 & 34.97 & 33.43 & 34.51 & $34 ., 0$ & 37.75 & 18.2 & \\
\hline wt: $343-510 \mathrm{C}$ & 23.45 & 26.83 & 26.41 & 25.69 & 32.43 & 31.13 & 26.21 & 27.84 & 24.31 & 21.51 & 31.5 & \\
\hline wt? $510+C$ & 44.02 & 37.75 & 22.62 & 25.73 & 20.73 & 17.00 & 16.65 & 15.91 & 11.14 & 9.39 & 34.2 & \\
\hline wt: Unc MAF coal & 7.09 & 5.67 & 3.50 & 3.67 & 3.13 & 2.81 & 3.42 & 2.94 & 2.47 & 2.22 & 3.7 & \\
\hline wt? total prod & 102.62 & 102.63 & 103.30 & 102.56 & 103.42 & 102.75 & 103.52 & $102.8^{\prime} 7$ & 103.42 & 103.75 & 102.4 & \\
\hline
\end{tabular}

*100\% Nitrogen Balance assumed to calculate NHI by disappearance of N fron liquid streans. 
Run No. 25 - 2:1 Lloydminster ResidiIllínols Coal No, 6

\subsection{1 wt? Mo Catalyst}

\begin{tabular}{|c|c|c|c|c|c|c|c|c|c|c|c|c|}
\hline Run & 25 & 25 & 25 & 25 & 25 & 25 & 25 & 25 & 25 & 25 & 19 Ave. & 21 Ave. \\
\hline Test Period & 6 & 7 & 11 & 13 & 17 & 21 & 23 & 27 & 31 & 41 & LTOS & $17-19$ \\
\hline \multicolumn{13}{|c|}{ Toluene Soluble Bottoas Product Properties: } \\
\hline TSB API & 2.3 & 2.1 & -2.5 & -1.2 & 0 & -0.8 & -4.3 & -5 & 0.7 & 0.4 & 3.3 & 7.4 \\
\hline TSB at: C & 85.92 & 85.71 & 86.62 & 86,84 & 86.48 & 87.38 & 87.84 & 86.57 & 86.91 & 86.88 & 85.98 & 88.3 \\
\hline TSB wt: H & 3.15 & 9.16 & 8.54 & 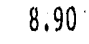 & 8.75 & 8.75 & 8.20 & 7.81 & 8.48 & 8.48 & 10.18 & 11.0 \\
\hline TSB nt? $S$ & 2.64 & 2.51 & 1.96 & 1.94 & 2.01 & 1.85 & 1.82 & 1.99 & 1.52 & 1.91 & 2.27 & 1.6 \\
\hline TSB wt: $N$. & 1.18 & 1.06 & 1.32 & 1.16 & 1.14 & 1.39 & 1.33 & 1.22 & 0.51 & 0.52 & NA & 1.6 \\
\hline TSB wt: HI & 36.34 & 24.31 & 25.52 & 21.89 & 19 & 21 & 59.34 & 31.48 & 8.12 & 12.56 & 11.65 & 4.9 \\
\hline TSB wt: KCRT & 27.69 & 23.76 & 30.50 & 27.93 & 25.23 & 26.3 & 31.98 & 32.90 & 19.24 & 20.01 & NA & 16.4 \\
\hline TSB wt: ash & 0.00 & 3.00 & 0.00 & 0,00 & 0 & 0 & 0 & 0 & 0 & 0 & 0.01 & 0.0 \\
\hline TSB pp》 Ho & 0 & 0 & 0 & 0 & 0 & 0 & 0 & 0 & 0 & 0 & 38 & 11.6 \\
\hline \multicolumn{13}{|l|}{ TSB D1160, vol\% } \\
\hline IBP, Deg C & 116 & 1.40 & 119 & 130 & 150 & 138 & 208 & 305 & 254 & 284 & 398.3 & 210.3 \\
\hline $5 \%$ & 480 & 444 & 475 & 458 & 361 & 350 & 454 & 451 & 352 & 350 & 457.3 & 467.0 \\
\hline $10 \%$ & 510 & 463 & 486 & 473 & 432 & 403 & 474 & 465 & 374 & 375 & 473.3 & 481.3 \\
\hline $20 \%$ & 539 & 495 & 502 & 492 & 470 & 473 & 495 & 497 & 400 & 399 & 493.0 & 504.0 \\
\hline $30 \%$ & $N A$ & 521 & 520 & 514 & 494 & 499 & $5 i 5$ & $52^{\prime} 3$ & 423 & 424 & 512.8 & 527.3 \\
\hline $40 \%$ & $\mathrm{NA}$ & $\mathrm{NA}$ & $N A$ & . 533 & 519 & $52 ?$ & NA & NA & 447 & 455 & $N A$ & $N A$ \\
\hline $50 \%$ & $N A$ & $N A$ & NA & $N A$ & $N A$ & NA & $N A$ & $N A$ & 472 & 192 & $N A$ & $N A$ \\
\hline $60 \%$ & $\mathrm{NA}$ & NA & NA & $N A$ & $N A$ & NA & Wh & NA & 506 & 525 & NA & $N A$ \\
\hline$E P$ & 550 & 539 & 535 & 533 & 532 & 530 & 530 & 540 & 533 & 547 & 542.8 & 539.7 \\
\hline \% QEP & 25 & 38 & 37 & 40 & 46 & 45 & 38 & 36 & 67 & 65 & $\mathbb{N A}$ & 36.3 \\
\hline Wt: D1160 OH & 22.98 & 36.12 & 35,60 & 38.31 & 46 & 45 & 35.76 & 35.08 & 66.85 & 6457 & 42.42 & 36.3 \\
\hline ISB OH API & 11.6 & 11.5 & 7.2 & 8.2 & 9.7 & 9.4 & 5.1 & 4 & 8 & 8.1 & 10.9 & 14.5 \\
\hline \multicolumn{13}{|l|}{ TSB OH GLC, wt? } \\
\hline IBP, Deg C & 111 & 110 & 111 & 111 & 119 & 110 & 195 & 250 & 200 & 177 & 390.3 & 252.7 \\
\hline $5 \%$ & 117 & 3.17 & 404 & 384 & 244 & 228 & 353 & 382 & 306 & 249 & 412.0 & 403.3 \\
\hline $10 \%$ & 396 & 105 & 433 & 420 & 302 & 286 & 409 & 411 & 332 & 275 & 434.5 & 431.7 \\
\hline $20 \%$ & 442 & 444 & 458 & 445 & 384 & 375 & 440 & 439 & 360 & 302 & 459.0 & 459.0 \\
\hline $30 \%$ & 468 & 463 & 472 & 460 & 423 & 419 & 456 & 457 & 378 & 320 & 474.8 & $47 \%, 0$ \\
\hline $40 \%$ & 484 & 475 & 481 & 472 & 444 & 444 & 468 & 471 & 395 & 337 & 488.8 & 487.0 \\
\hline $50 \%$ & 499 & 486 & 492 & 481 & 460 & 462 & 477 & 481 & 411 & 355 & 500.3 & 497.7 \\
\hline $60 \%$ & 511 & 499 & 503 & 492 & 473 & 475 & 487 & 494 & 426 & 373 & 511.8 & 508.0 \\
\hline $70 \%$ & 524 & 512 & 514 & 505 & 485 & 488 & 500 & 507 & 442 & 392 & 523.5 & 519.3 \\
\hline $80 \%$ & 535 & 527 & 528 & 520 & 502 & 505 & 514 & 522 & 461 & 414 & 536.5 & 531.0 \\
\hline $90 \%$ & 552 & 544 & 543 & 538 & 527 & 527 & 532 & 538 & 484 & 440 & 554.5 & 545.3 \\
\hline $95 \%$ & 570 & 561 & 560 & 553 & 544 & 543 & 545 & 550 & 506 & 463 & 568.5 & 559.7 \\
\hline EP & 570 & 574 & 573 & 566 & 573 & 567 & 573 & 574 & 546 & 507 & 577.5 & 572.0 \\
\hline$\%$ QEP & 95 & 97 & 97 & 97 & 98 & 98 & 99 & 99 & 100 & 100 & 100.0 & 97.3 \\
\hline wt: D1160 Bt Bts & 77.02 & 63.88 & 64,40 & 61.69 & 54 & 55 & 64.24 & 64.92 & 33.15 & 35.43 & 57.6 & 63.7 \\
\hline TSB Btns API & 2.3 & -1.5 & -8.8 & -8.3 & -8.9 & -10.2 & -11.4 & -21.2 & -12.8 & -12.6 & -4.0 & 5.7 \\
\hline
\end{tabular}


Run No, 25 - 2:1 Lloydminster ResidiIllinois Coal No. 6

\subsection{1 wt? No Catalyst}

Run

Test Period $\begin{array}{rrrr}25 & 25 & 25 & 25 \\ 6 & 7 & 11 & 13\end{array}$

Overhead Product Properties:

OH API
OH wt\% C
OH wt\% H
OH wt\% S
Oil wt\% N
OH GLC, wt\%
IBP, Deg.C.
$5 \%$
$10 \%$
$20 \%$
$30 \%$
$40 \%$
$50 \%$
$60 \%$
$70 \%$
$80 \%$
$90 \%$
$85 \%$
EP.

$$
23.2
$$

32.23

10.99

23.7

83.91

\section{$26.9 \quad 30.7$}

$81.31 \quad 83.44$

1.45

0.471

11,11

11.03

11.64

0.99

$0.459 \cdot 0.469$

0.455

$\begin{array}{rrr}20 & 23 & -16 \\ 113 & 115 & 37 \\ 185 & 186 & 87\end{array}$

$\begin{array}{rrr}-38 & 146 & 152 \\ 34 & 194 & 199 \\ 64 & 215 & 219 \\ 159 & 245 & 248 \\ 224 & 270 & 272 \\ 259 & 294 & 296 \\ 291 & 316 & 318 \\ 320 & 340 & 342 \\ 349 & 363 & 365 \\ 378 & 387 & 391 \\ 409 & 415 & 421 \\ 428 & 432 & 439 \\ 465 & 466 & 496 \\ 100 & 100 & 100\end{array}$

$20.6: 19.6$

$\begin{array}{lll}86.6 & 87.38\end{array}$

$11.2 \quad 10.82$

1.06

0.98

0.625

$185 \quad 186$

$237 \quad 236$

$273 \quad 269$

$305 \quad 299$

$334 \quad 326$

$363 \quad 353$

$389-379$

$417 \quad 406$

$445 \quad 432$

$465 \quad 448$

$494 \quad 489$

$100 \quad 100$

196

$$
239
$$

271

302

331

361

390

423

443

475

\% PEP sk

Total Liquid Properties

nt: C

wt: $\mathrm{H}$

wt? 5

wt: $N$

wt? HI

wt: MCRT

wto dist. (371C-)
$11.0 \quad 10.9$

84.4

$9.9 \quad 10.0$

$2.1 \quad 2.1$

$0.9 \quad 0.8$

$21.1 \quad 14$.

$16.1 \quad 14.1$

26.9 .28 .1
16.5

83.2

10.2

$17.4 \quad 12.9$

$84.9 \quad 86.6$

$10.5 \quad 10.3$

$1.4 \quad 1.4 \cdot 1.4$

0.8

0.8

9.1

11.7

10.8

47.9 .

\section{1}

87.4

10.2

16.7
85.9

13.3

20.6

19.2

$\begin{array}{ll}21.4 & 84.4\end{array}$

$10.4 \quad 11.1$

10.9

1.2

1.2

0.9

1.3

0.9

0.7

0.7

0.4

0.4

16.3

B. 3

3.1

3.2

$8.4 \quad 8.8$

8.7
54.1

62.4

5.0

69.3

\begin{tabular}{|c|c|c|c|c|c|c|c|c|c|c|c|c|}
\hline UCC wt: C & 86.4 & 86.4 & 84.4 & 83.7 & 84.0 & 82.9 & 83.4 & 83.7 & 82.9 & 83.6 & $N A$ & 82.2 \\
\hline UCC wt? H & 6.0 & 5.9 & 5.4 & 5.6 & 5.7 & 5.6 & 5.3 & 5.2 & 5.6 & 4.8 & $N A$ & 5.7 \\
\hline UCC wt: S & 5.5 & 5.7 & 8.3 & 8.9 & 8.3 & 9.9 & 9.0 & 9.9 & 10.7 & 11.0 & NA & 10.2 \\
\hline UCC wt? N & 2.1 & 2.1 & 1.9 & 1.8 & 2.1 & 1.6 & 2.3 & 1.3 & 0.8 & 0.7 & $N A$ & 1.8 \\
\hline DesuI & 56.19 & 59.01 & 73.71 & 73.75 & 70.87 & 76.97 & 78.05 & 77.20 & 81.85 & 76.48 & 64.6 & 76.7 \\
\hline \& Denitrif. $\star \star * *$ & 23.51 & 31.27 & 36.80 & 39.98 & 32.84 & 32.66 & 42.72 & 45.75 & 65.61 & 67.91 & NA & 22.3 \\
\hline
\end{tabular}

Unconverted Coal Solids Properties $\star$ :

* *

** calculated as ( $g$ TIB $x) /(g$ TIB $C+g$ TIB H $+g$ TIB $S-g$ Cat $S+g$ TIB N)*100\%

***Calculated as "(Feed S or N - Liguid Prod. $S$ or N)/(Feed S or N)*100\%" 


\section{MAF Coal Conversion vs. Temperature}

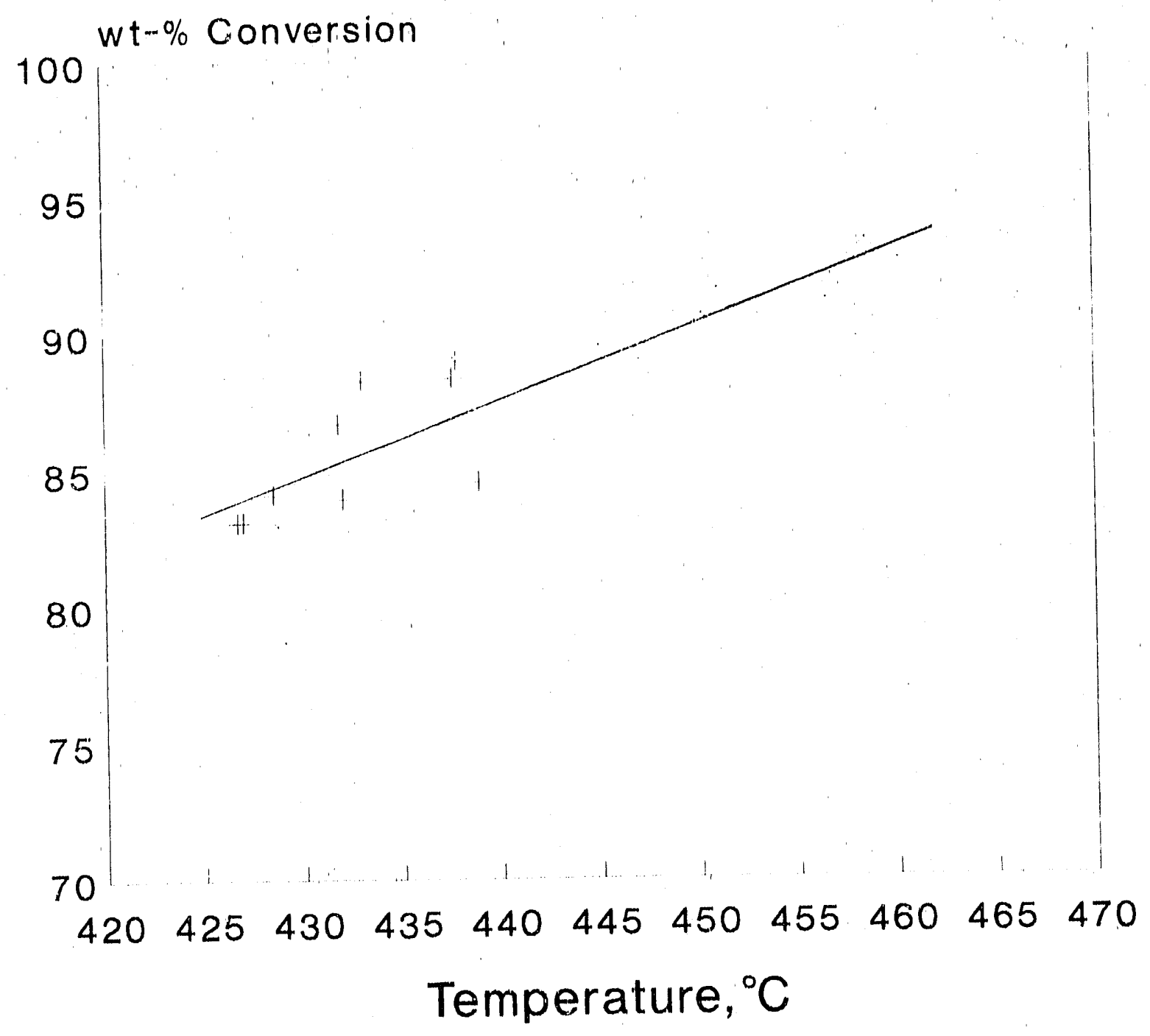

I Run $24-0.13 \%$ Mo

Run $25-0.11 \%$ Mo

K6.7 Catalyst, C1.4 Coal, R10 Resid

3000 psig, base WHSV, 2:1 Resid:Coal 


\section{Nondistillable Conversion vs. Temperature}

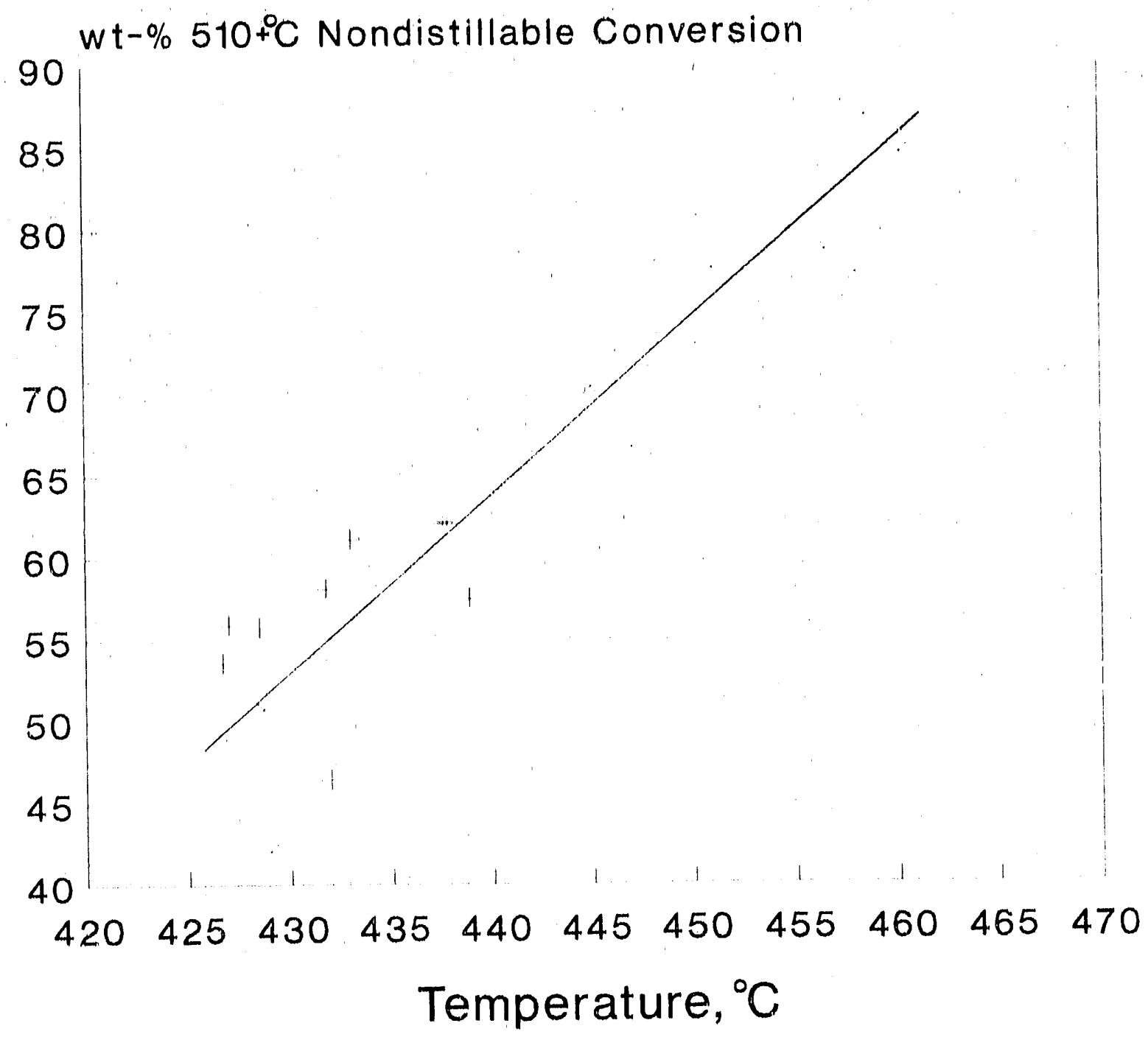

Run $24-0.13 \%$ Mo

Run $25-0.11 \%$ Mo

K6.7 Catalyst, C1.4 Coal, R10 Resid

3000 psig, base WHSV, 2:1 Resid:Coal 


\section{Hept. Insol. Conversion vs. Temperature}

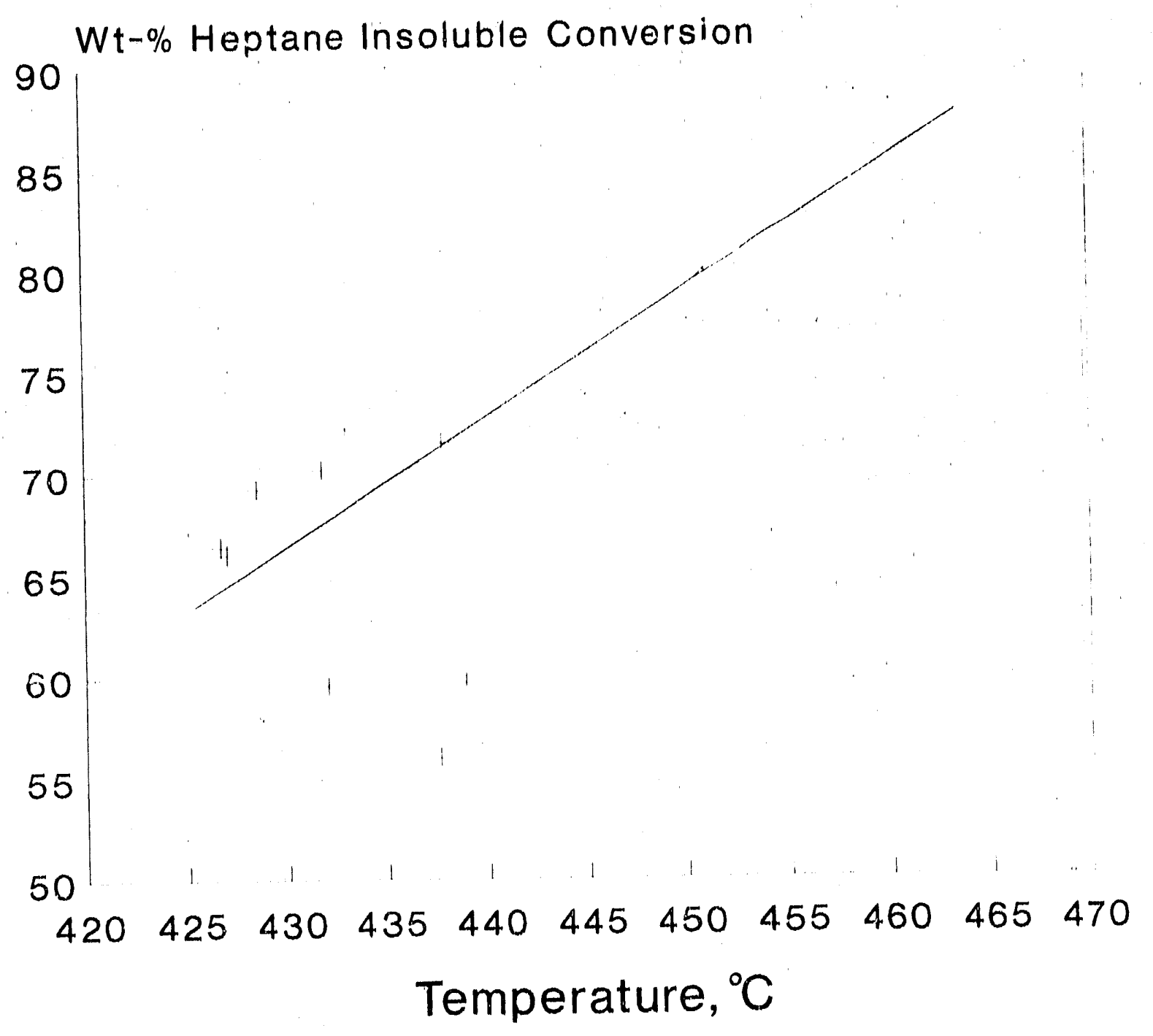

Run $24-0.13 \%$ Mo

Run $25-0.11 \%$ Mo

K6.7 Catalyst, C1.4 Coal, R10 Resid

$3000 \mathrm{psig}$, base WHSV, 2:1 Resid:Coal 


\section{$371^{\circ} \mathrm{C}+$ ND Conversion vs. Temperature}

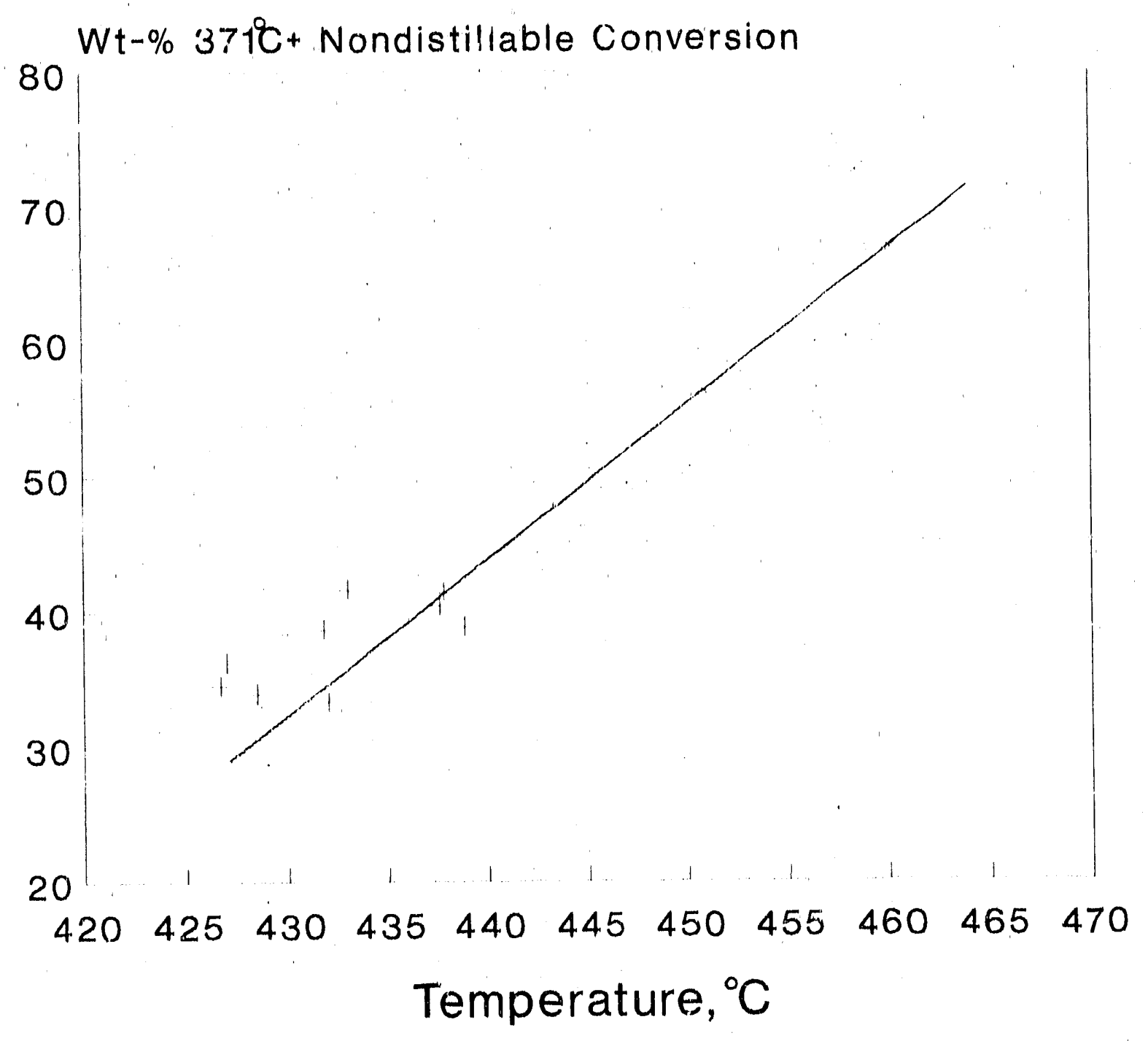

1 Run $24-0.13 \%$ Mo Run $25-0.11 \%$ Mo

K6.7 Catalyst, C1.4 Coal, R10 Resid

3000 psig, base WHSV, 2:1 Resid:Coal

$$
\text { C-4 }
$$




\section{Wt-\% H2 Consumption vs. Temperature}



Run $24-0.13 \%$ Mo Run $25-0.11 \%$ Mo

K6.7 Catalyst, C1.4 Coal, R10 Resid

3000 psig, base WHSV, 2:1 Resid:Coal 


\section{Light Ends Yields vs. Temperature}

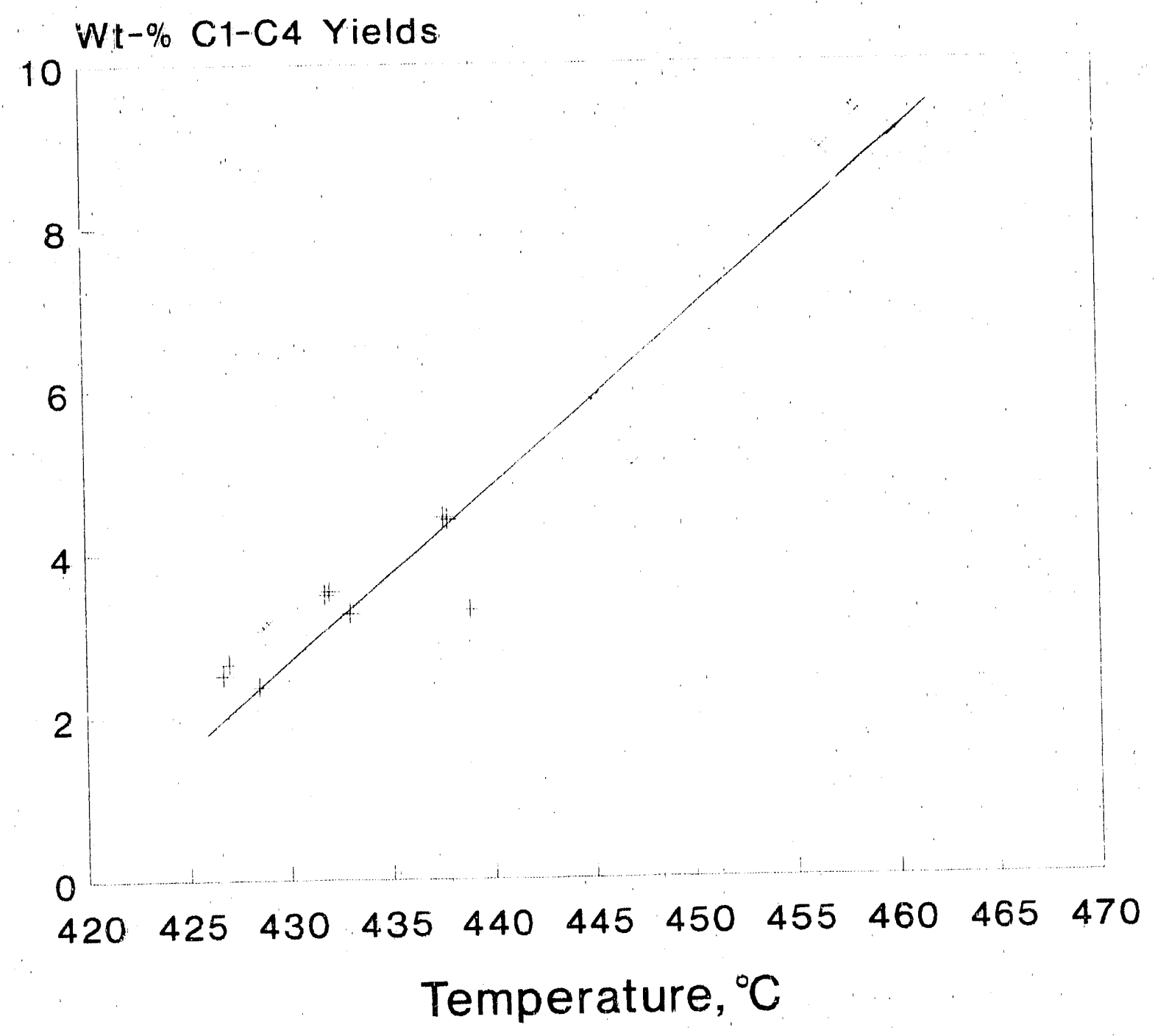

+ Run $24-0.13 \%$ Mo Run $25-0.11 \%$ Mo

K6.7 Catalyst, C1.4 Coal, R10 Resid 3000 psig, base WHSY, 2:1 Resid.Coal 


\section{C5-177 C Naphtha Yield vs. Temperature}

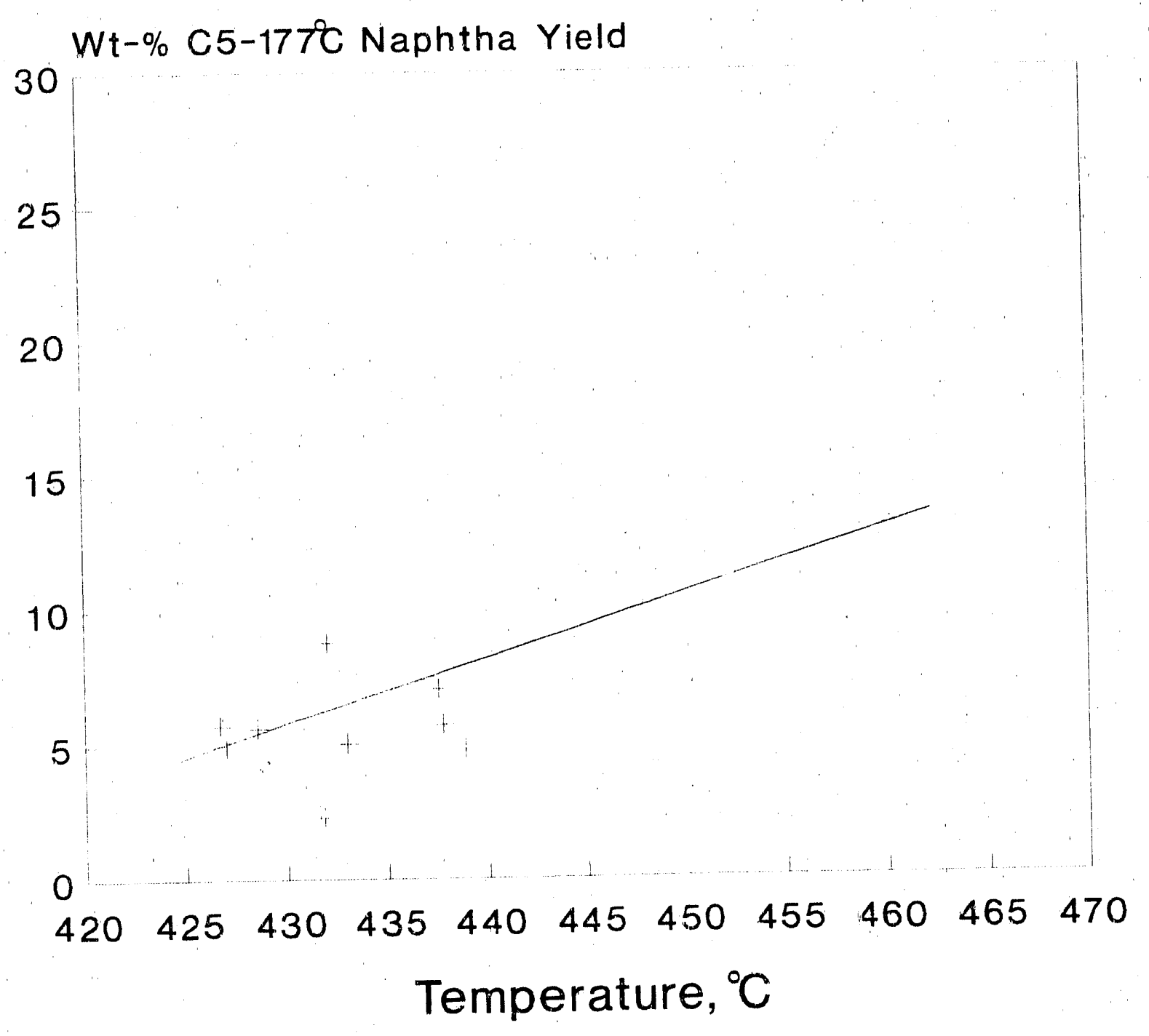

Run $24-0.13 \%$ Mo Run $25-0.11 \%$ Mo

K6.7 Catalyst, C1.4 Coal, R10 Resid

3000 psig, base WHSV, 2:1 Resid:Coal 


\section{$177-343^{\circ} \mathrm{C}$ Dist. Yield vs. Temperature}

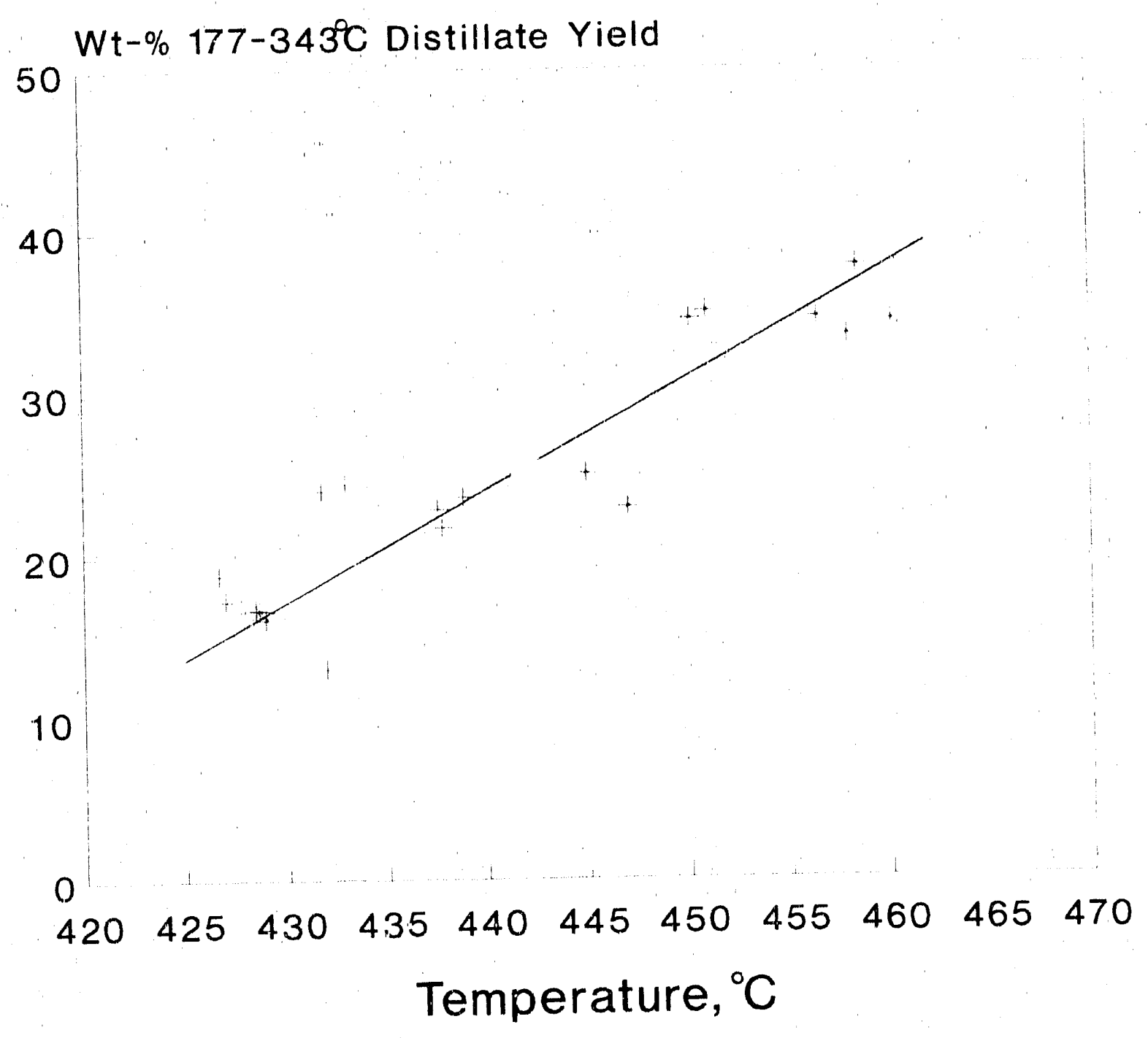

Run $24-0.13 \%$ Mo Run $25-0.11 \%$ Mo

K6.7 Catalyst, C1.4 Coal, R10 Resid

3000 psig, base WHSV, 2:1 Resid:Coal 


\section{3-510 $\mathrm{C}$ VGO Yield vs. Temperature}

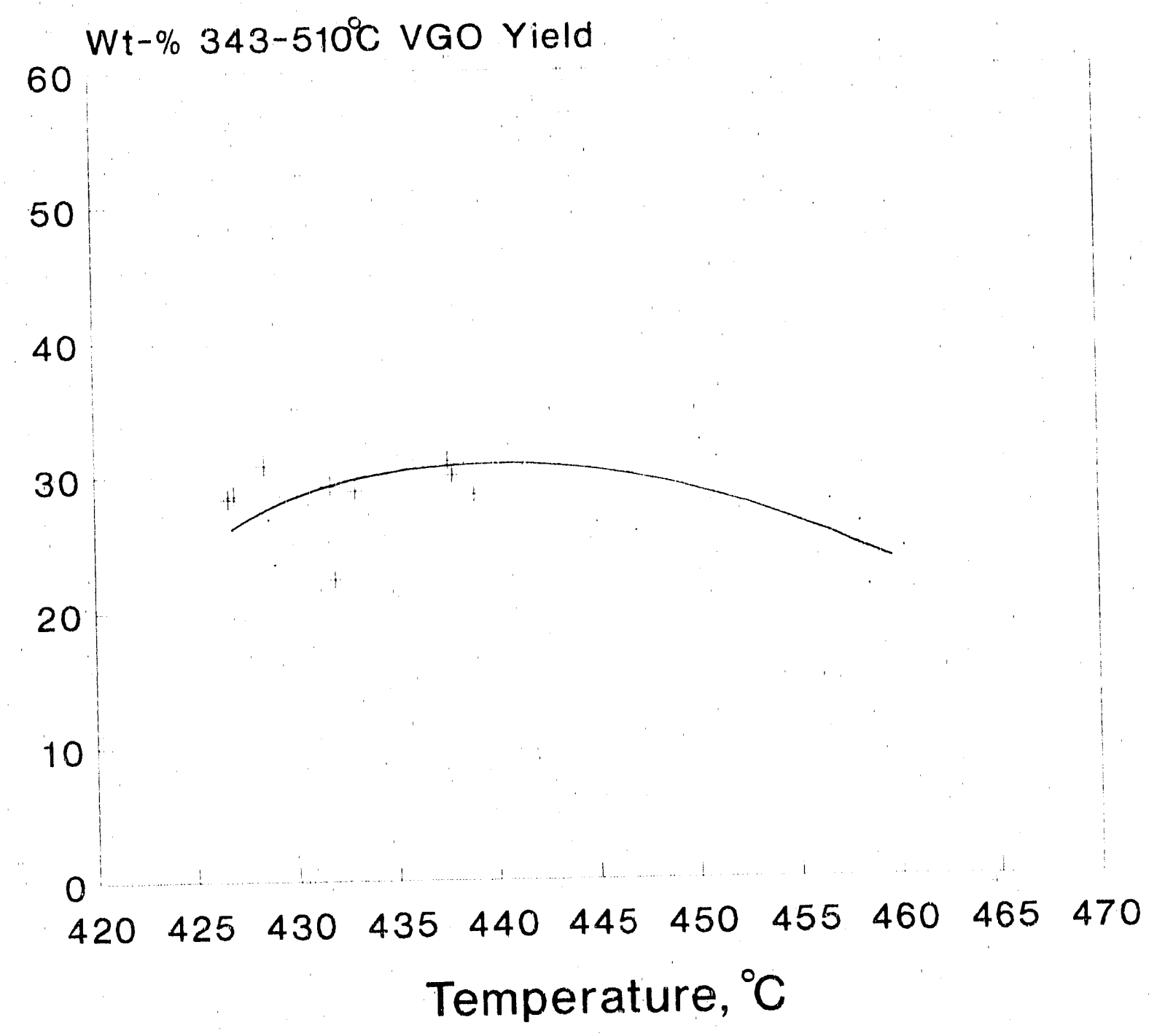

Run $24-0.13 \%$ Mo Run $25-0.11 \%$ Mo

K6.7 Catalyst, C1.4 Coal, R10 Resid

3000 psig, base WHSV, 2:1 Resid:Coal 


\section{$510^{\circ} \mathrm{C}+\mathrm{ND}$ Yield vs Temperature}

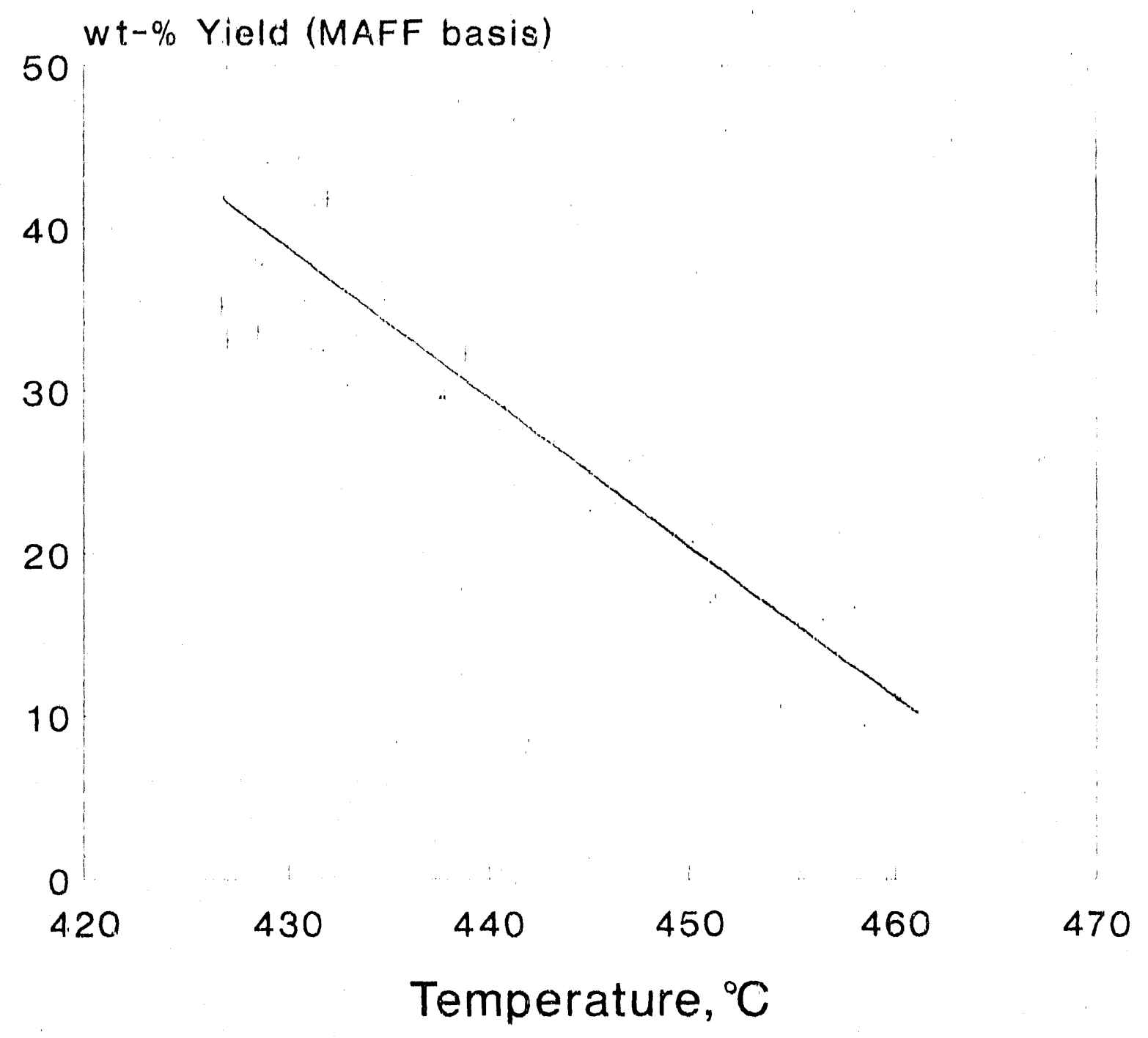

+ Run $24-0.13 \%$ Mo

Run $25-0.11 \%$ Mo

K6.7 Catalyst, C1.4 Coal, R10 Resid

3000 psig, base WHSV, 2:1 Resid:Coal 


\section{C5-510 $\mathrm{C}$ Liquid Yield vs. Temperature}

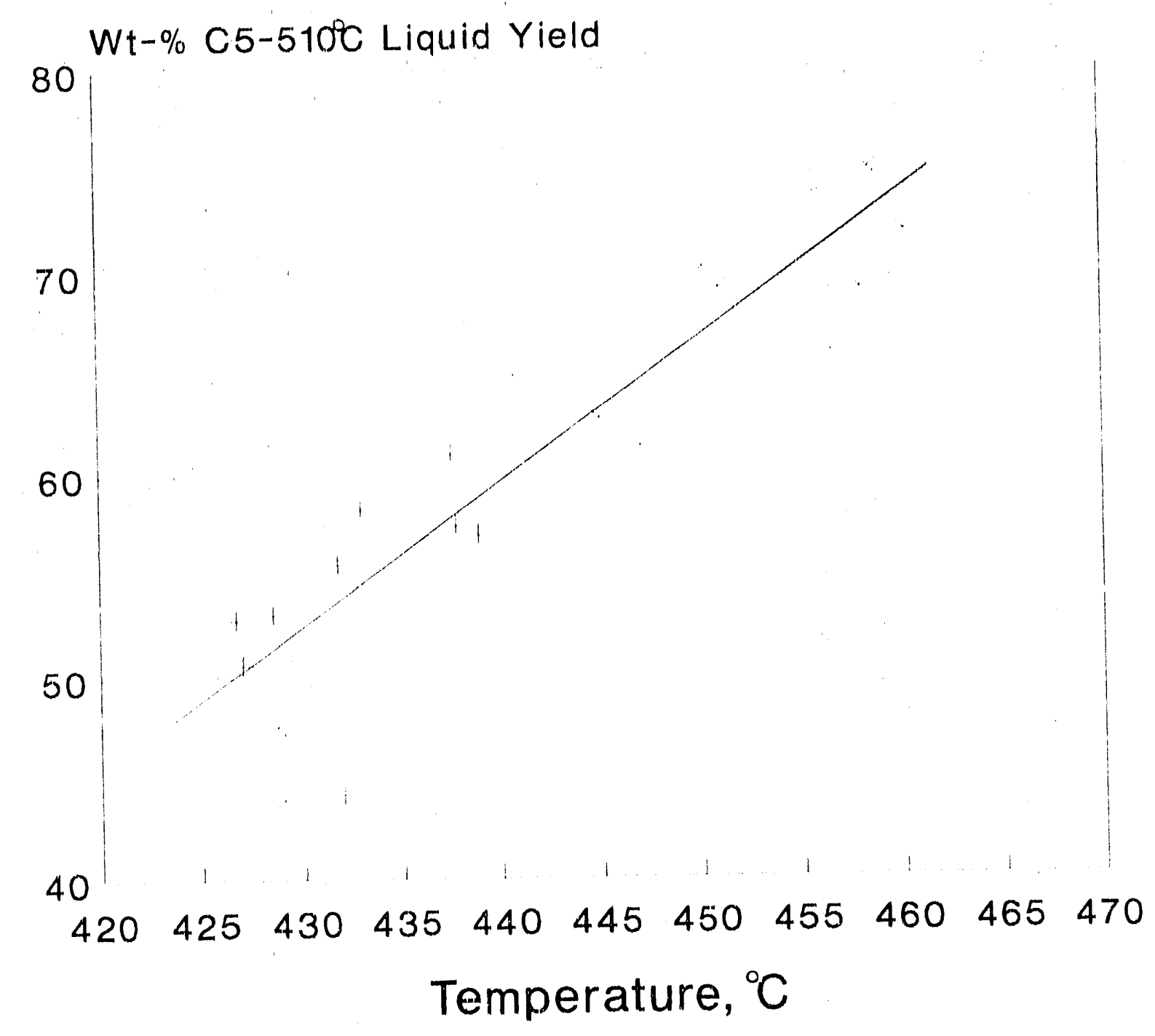

K6.7 Catalyst, C1.4 Coal, R10 Resid 3000 psig, base WHSV, 2:1 Resid:Coal 


\section{C5+ Total Liquid Yield vs. Temperature}

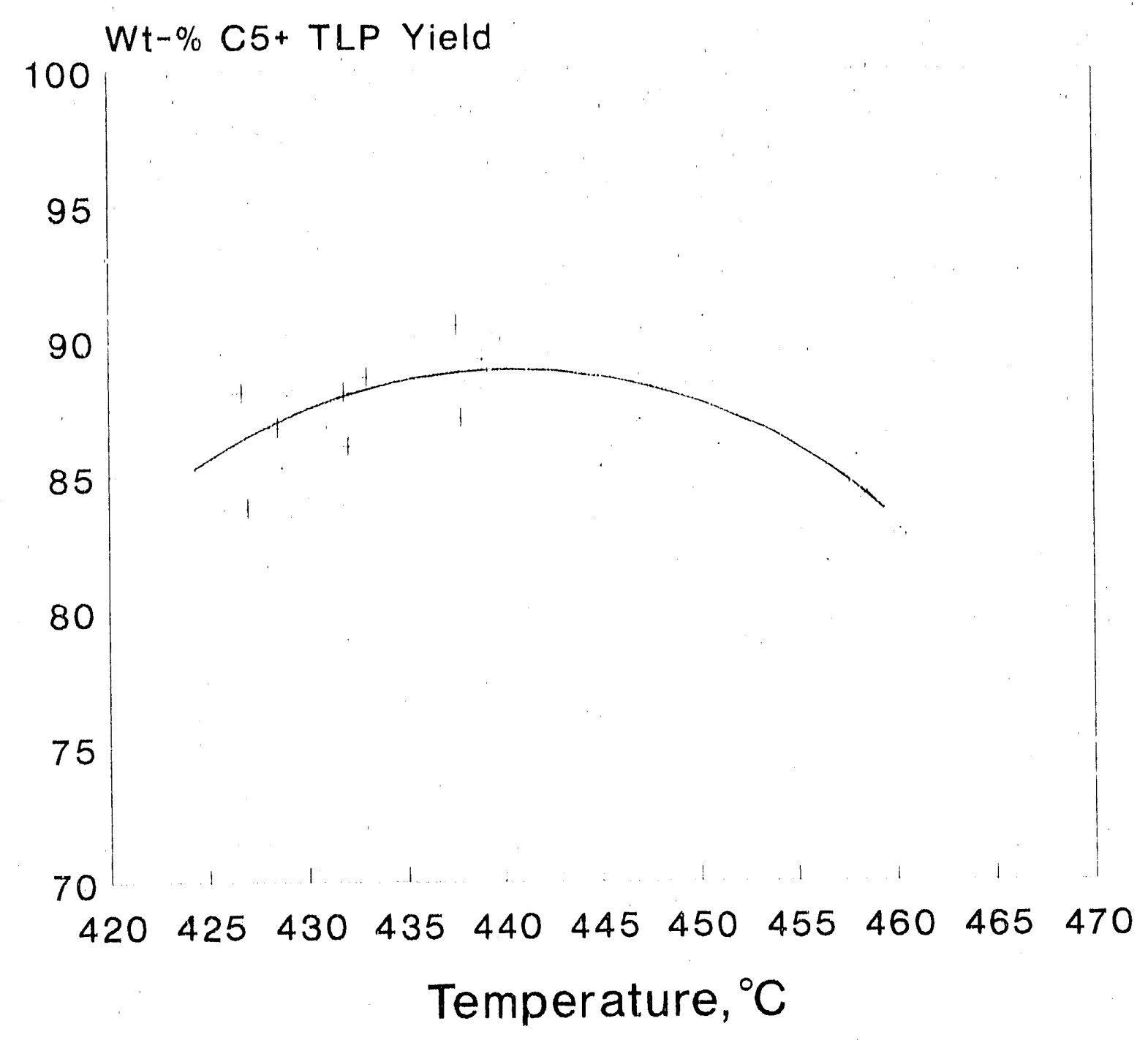

Run $24-0.13 \%$ Mo Run $25-0.11 \%$ Mo

K6.7 Catalyst, C1.4 Coal, R10 Resid

3000 psig, bast WHSV, 2:1 Resid:Coal 


\section{C5+ Liquid Product API vs. Temperature}

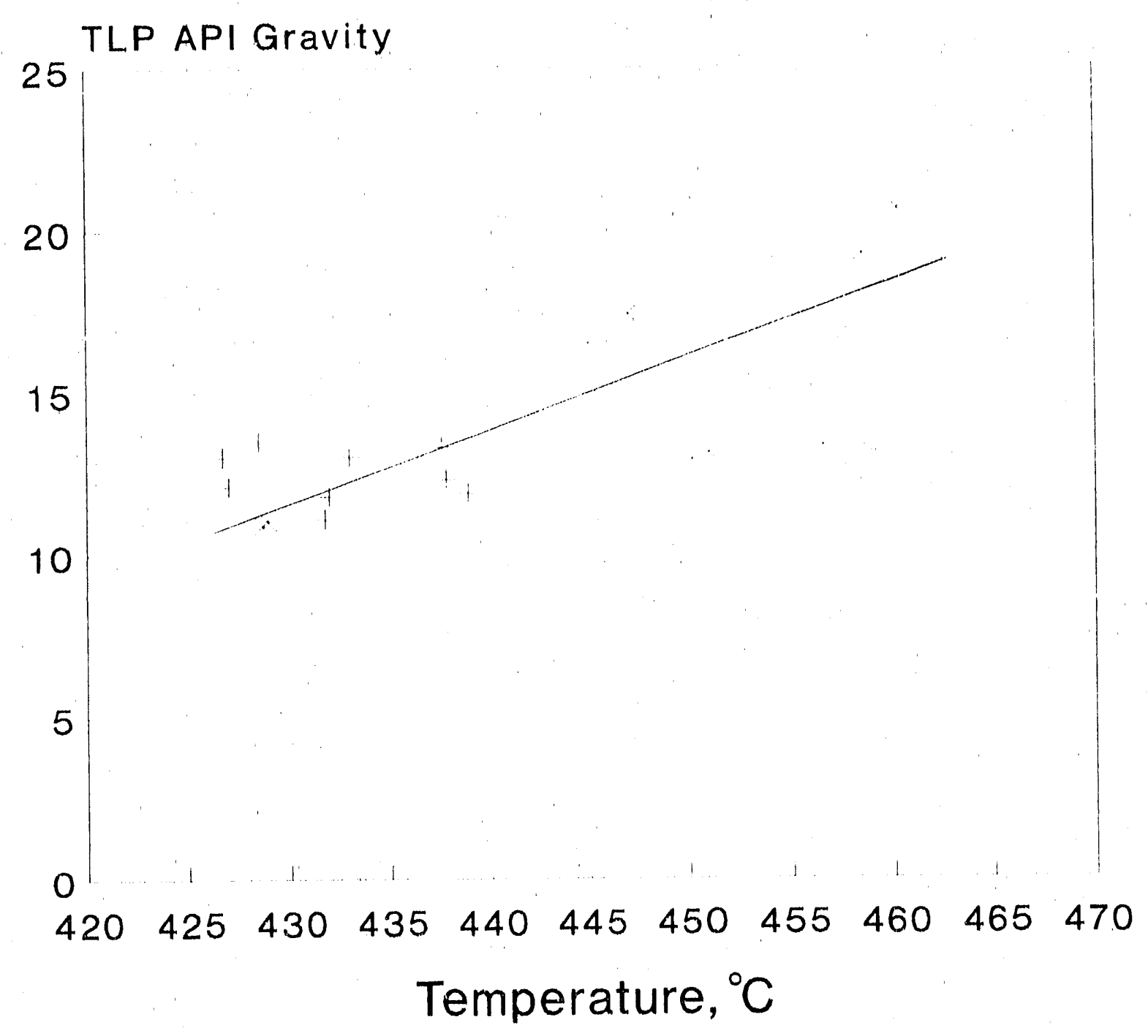

Run $24-0.13 \%$ Mo Run $25-0.11 \%$ Mo

K6.7 Catalyst, C1.4 Coal, R10 Resid

3000 psig, base WHSV, 2:1 Resid:Coal 


\section{C5+ Product Wt-\% S vs. Temperature}

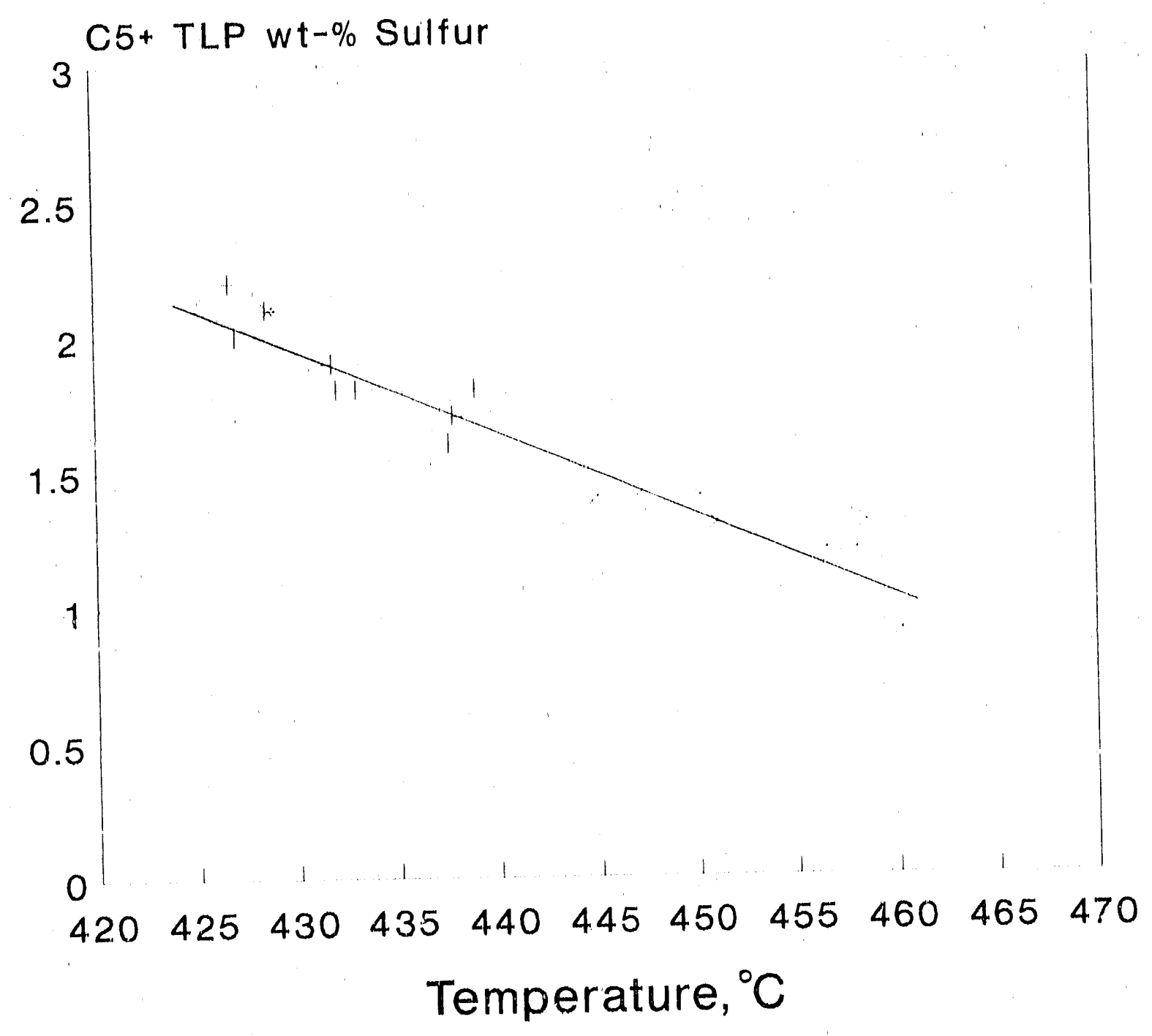

1. Run $24-0.13 \%$ Mo Run $25-0.11 \%$ Mo

K6.7 Catalyst, C1.4 Coal, R10 Resid

3000 psig, base WHSV, 2:1 Resid:Coal 


\section{C5+ Product Wt-\% N vs. Temperature}

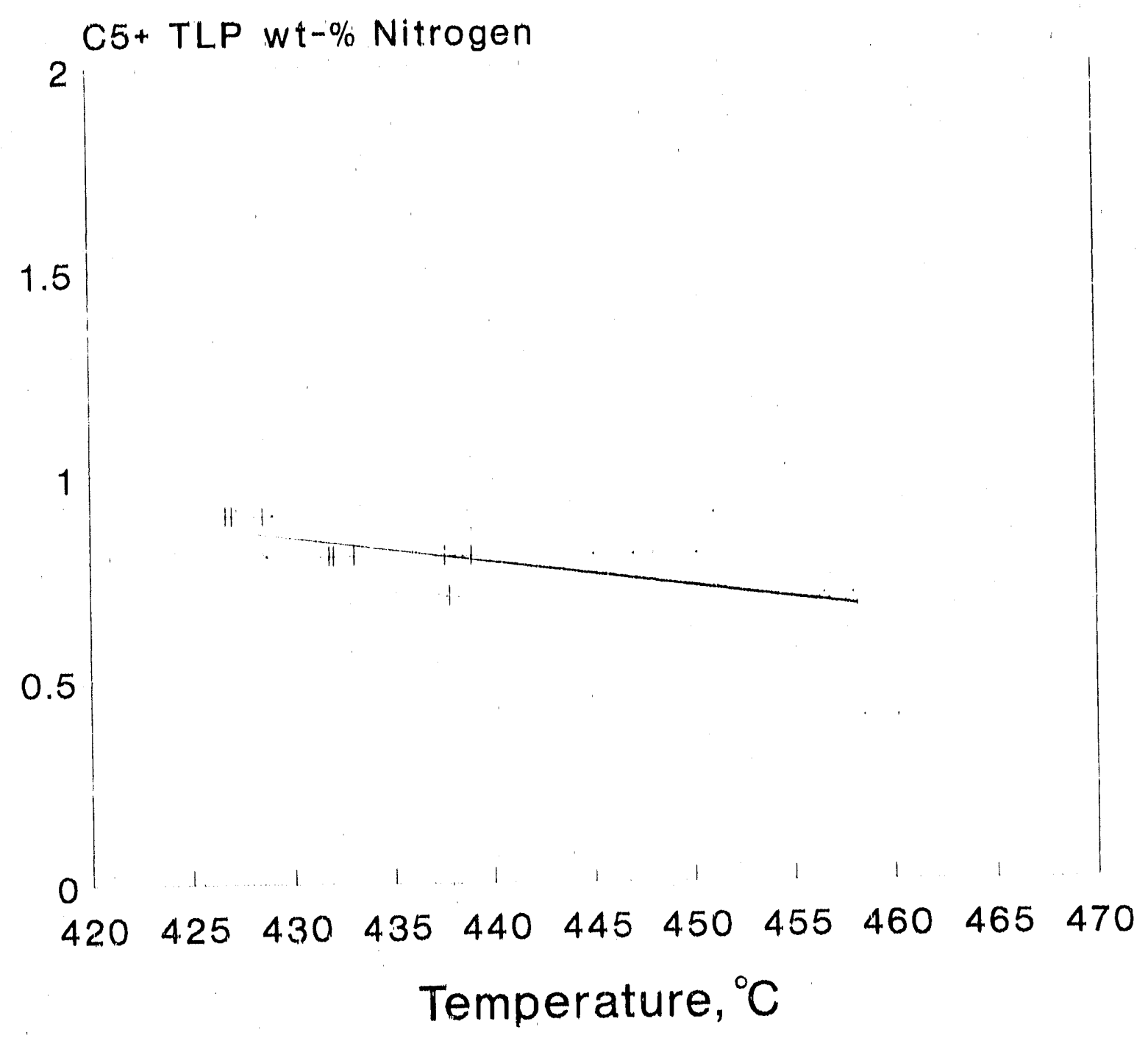

+ Run $24-0.13 \%$ Mo Run $25-0.11 \%$ Mo

K6.7 Catalyst, C1.4 Coal, R10 Resid

$3000 \mathrm{psig}$, base WHSV, 2:1 Resid:Coal 


\section{$\mathrm{C} 5+$ Product $\mathrm{Wt}-\% \mathrm{HI}$ vs. Temperature}

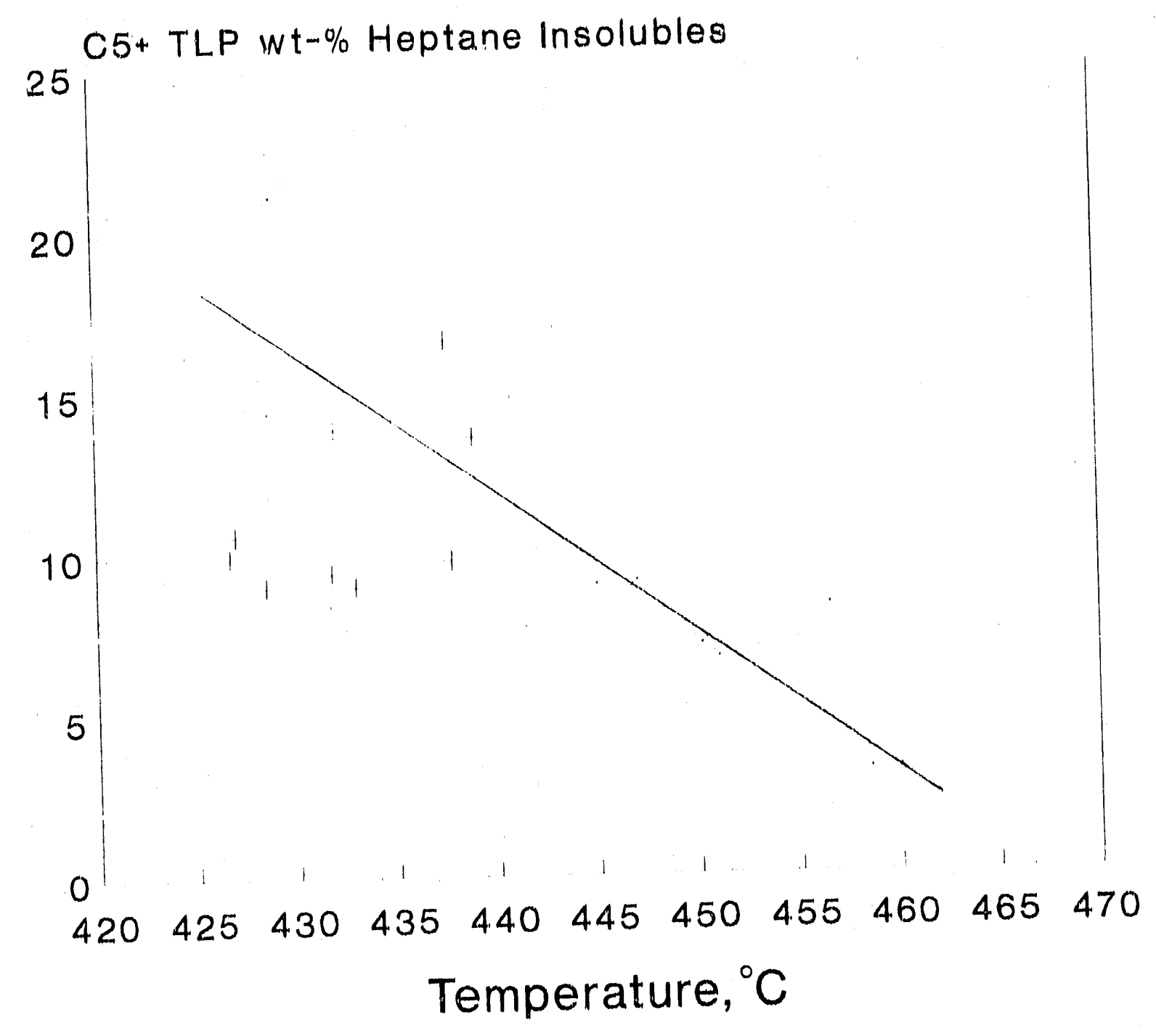

I Run $24-0.13 \%$ Mo

Run $25-0.11 \%$ Mo

K6.7 Catalyst, C1.4 Coal, R10 Resid

3000 psig, base WHSV, 2:1 Resid:Coal 


\section{C5+ Product $\mathrm{Wt} \% \mathrm{MCRT}$ vs. Temperature}

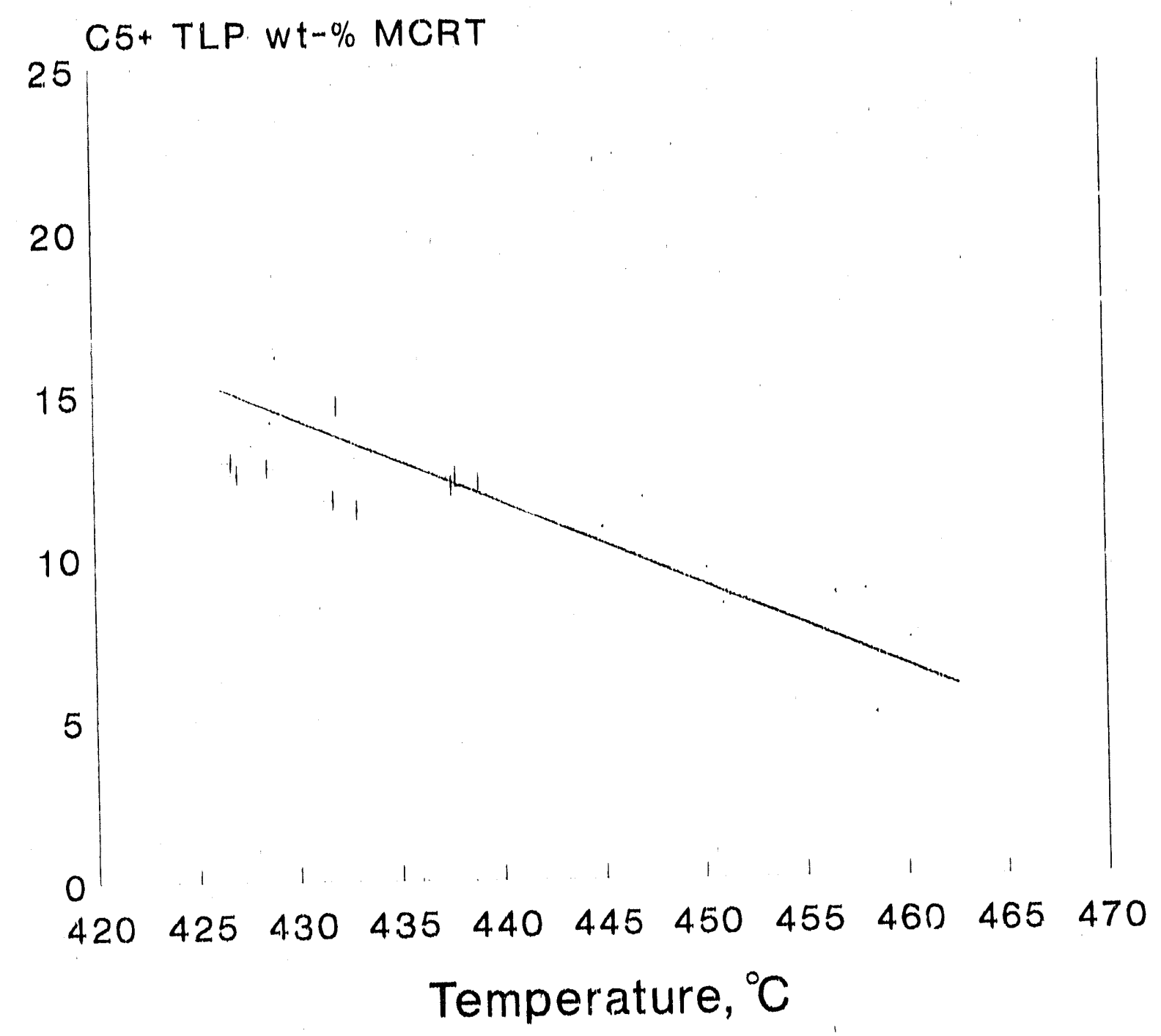

Run $24-0.13 \%$ Mo

Run $25 \cdot 0.11 \%$ Mo

K6.7 Catalyst, C1.4 Coal, R10 Resid

3000 psig, base WHSV, 2:1 Resid:Coal 


